\title{
Utilizing cosmic-ray positron and electron observations to probe the averaged properties of Milky Way pulsars
}

\author{
Ilias Cholis 1, * and Iason Krommydas ${ }^{2}, \dagger$ \\ ${ }^{1}$ Department of Physics, Oakland University, Rochester, Michigan, 48309, USA \\ ${ }^{2}$ Physics Division, National Technical University of Athens, Zografou, Athens, 15780, Greece
}

(Dated: November 12, 2021)

\begin{abstract}
Pulsars have long been studied in the electromagnetic spectrum. Their environments are rich in high-energy cosmic-ray electrons and positrons likely enriching the interstellar medium with such particles. In this work we use recent cosmic-ray observations from the AMS-02, CALET and $D A M P E$ collaborations to study the averaged properties of the local Milky Way pulsar population.

We perform simulations of the local Milky Way pulsar population, for interstellar medium assumptions in agreement with a range of cosmic-ray nuclei measurements. Each such simulation contains $\sim 10^{4}$ pulsars of unique age, location, initial spin-down power and cosmic-ray electron/positron spectra. We produce more than $7 \times 10^{3}$ such Milky Way pulsar simulations. We account for and study i) the pulsars' birth rates and the stochastic nature of their birth, ii) their initial spin-down power distribution, iii) their time evolution in terms of their braking index and characteristic spindown timescale, iv) the fraction of spin-down power going to cosmic-ray electrons and positrons and v) their propagation through the interstellar medium and the Heliosphere. We find that pulsars of ages $\sim 10^{5}-10^{7} \mathrm{yr}$, have a braking index that on average has to be 3 or larger. Given that electromagnetic spectrum observations of young pulsars find braking indices lower than 3, our work provides strong hints that pulsars' braking index increases on average as they age, allowing them to retain some of their rotational energy. Moreover, we find that pulsars have relatively uniform properties as sources of cosmic-ray electrons and positrons in terms of the spectra they produce and likely release $\mathrm{O}(10 \%)$ of their rotational energy to cosmic-rays in the ISM. Finally, we find at $\simeq 12$ $\mathrm{GeV}$ positrons a spectral feature that suggests a new subpopulation of positron sources contributing at these energies.
\end{abstract}

\section{INTRODUCTION}

Pulsars represent a class of energetic sources whose properties have been probed over more than 50 years via observations in the electromagnetic spectrum. Emission from pulsars and their environments has been detected in the radio, 1 18 , infrared and visible 9 13, ultraviolet [14, 15], X-rays, 12, 16, 20, gamma-rays [21 26] and most recently, a clear detection of powerful Milky Way pulsars at $O(10) \mathrm{TeV}$ gamma-rays has been established [27 31. Most of the observed photons from pulsars and their surrounding pulsar wind nebulae $(\mathrm{PWNe})$-where those are present- originate from cosmic-ray electrons and positrons and are emitted via curvature radiation [32, 33, synchrotron radiation [24, 34, 35] and at the highest energies inverse Compton emission [24, 36 38]. The fact that we have observed $O(10) \mathrm{TeV}$ gamma-rays from certain pulsars that are still surrounded by their respective PWN clearly sets a lower limit on the electron and positron cosmic-ray energies in these environments. We expect that such pulsars will act as sources of cosmicray electrons and positrons that are released into the interstellar medium (ISM). In fact, we expect for electrons and positrons to be further accelerated as they propagate through the termination shock of the respective PWNe before entering the ISM [39, 40]. If Milky Way pulsars are

\footnotetext{
* cholis@oakland.edu, ORCID: orcid.org/0000-0002-3805-6478

$\dagger$ iason.krom@gmail.com
}

prominent sources of high-energy cosmic-ray electrons and positrons then we could expect to see their contribution to the relevant cosmic-ray measurements and most notably in the cosmic-ray positron flux spectrum.

Cosmic-ray positrons are produced in inelastic collisions of high-energy cosmic-ray nuclei with the ISM gas and are typically referred to as secondary positrons. In the same type of interactions matter cosmic-ray secondary electrons and secondary nuclei as Boron are produced. Those have been modeled in [41-49] and are in agreement with the current observations [50 52]. A prominent exception is the spectrum of the positron fraction $e^{+} /\left(e^{+}+e^{-}\right)$, measured by $[53,56$ to rise above 5 $\mathrm{GeV}$, in disagreement with the expectation from same type of models. This suggests an additional source of high-energy cosmic-ray positrons. Such positrons can come from near-by Milky Way pulsars [40, 57-72]. One alternative to pulsars includes local and recent supernova remnants (SNRs) [73 83]. However, given that SNRs are the major source of all cosmic rays, in order to explain the rising positron fraction, the metallicities of environments of recent and close-by SNRs have to be different from those of the Milky Way on average [79, 80, 84, 85]. Another possibility is that of particle dark matter [66, 86, 105]. While such particle dark matter models have been constrained by cosmic-microwave background (CMB) data [106-111] and $\gamma$-rays [112 114, the full parameter space has not been entirely excluded.

In this paper we are going to use the cosmic-ray observations from the Alpha Magnetic Spectrometer ( $A M S$ - 
02), the Calorimetric Electron Telescope (CALET) onboard the International Space Station and the DArk Matter Particle Explorer (DAMPE) satellite. We are going to set constraints on the contribution of local Milky Way pulsars to the electron and positron $\left(e^{ \pm}\right)$fluxes and in turn probe their averaged properties. For cosmic-ray energies $E$ above $10 \mathrm{GeV}$ the propagation of electrons and positrons is mostly affected by their energy losses due to synchrotron radiation and inverse Compton scattering [115. The relevant energy loss timescale for electrons and positrons of initial energy $E_{\text {init }}$ to lose half its energy is,

$$
\tau_{\text {loss }}\left(E_{\text {init }}\right) \simeq 20 \times\left(\frac{E_{\text {init }}}{10 \mathrm{GeV}}\right)^{-1} \mathrm{Myr} .
$$

Pulsars lose their rotational kinetic energy within $O(10)$ kyr. Roughly that is also equal to the time that magnetic fields in the surrounding PWNe and the further out SNR become weak enough to allow the relevant cosmic-ray $e^{ \pm}$ to effectively escape. The $O(10) \mathrm{kyr}$ timescale is typically one to four orders of magnitude smaller by comparison to the timescale cosmic-rays need to reach us via diffusion from $O(100) \mathrm{pc}-O(1) \mathrm{kpc}$ distances where most pulsars are at. Thus pulsars can be treated as approximately injecting an appreciable fraction of their rotational energy to cosmic-ray electrons and positrons at the beginning of their existence [40, 62, 1

A result of Eq. 11, is that as the observed energy of cosmic rays increases, the number of potential sources drops given that only most recent pulsars have an age that is similar to $\tau_{\text {loss. }}$ As pulsars are born in the Milky Way with a rate of $\simeq 1$ per century [117-121, only a small number of them can contribute, and only from an increasingly smaller distance. The relation connecting the maximum energy that electrons and positrons can have originating from a distance $R$ was approximated in 122 to be $E_{\max } \sim 100 \mathrm{GeV}(R / 2 \mathrm{kpc})^{-2}$. For instance at 500 $\mathrm{GeV}$ we are probing only pulsars from within $\sim 400 \mathrm{pc}$. As there is only a small number of such pulsars, the discreteness of those sources will result in subsequent features [40, 62, 122, 123, (see also 83] for a similar study on the impact of recent SNRs). With the recent refined observations by $A M S-02$ and the observations by CALET and $D A M P E$ that extend up to $5 \mathrm{TeV}$ we will probe the properties of these pulsars. The lower energies of 5-500 $\mathrm{GeV}$, are also used and provide us with valuable information on the averaged properties of pulsars that are now "middle aged" and of up to $\mathrm{O}(10) \mathrm{Myr}$ and are located within $4 \mathrm{kpc}$. Finally, we will show that we can also

\footnotetext{
${ }^{1}$ A recent work on pulsars surrounded by $\mathrm{TeV}$ halos, has suggested that some pulsars may be able to contain their cosmic rays well after their birth [116]. As long as the injection phase of GeV$\mathrm{TeV}$ cosmic rays from these environments into the ISM occurs on a timescale significantly smaller than their propagation time, our basic analysis is not changed as this would be on average an overall time-shift for the pulsar population.
}

asses information on the properties of the ISM within that same volume.

In section III we discuss the simulations that we perform to account for astrophysical uncertainties on a) the stochastic birth distribution in space and time of the pulsar source-population, b) the initial properties of the total energy output of these sources, c) their timeevolution, d) their injection spectral properties of cosmicray $e^{ \pm}$and e) the propagation of cosmic rays through the ISM and the Heliosphere. Then in section III, we will discuss the data that we use and our fitting procedure. In section IV] we present our results. We show first our results from comparing to the observations above 15 $\mathrm{GeV}$ and then further discuss the lower energy analysis of measurements down to $5 \mathrm{GeV}$ in the positron fraction and flux, where we notice a somewhat significant feature at $\simeq 12 \mathrm{GeV}$. Finally, we give our conclusions and summary in section $\mathrm{V}$.

\section{MILKY WAY PULSAR SIMULATIONS}

Our pulsar simulations account for,

- the stochastic nature of the neutron stars' birth distribution in space and in time. We run simulations for different birth rates of neutron stars,

- the initial conditions of the neutron stars in terms of their initial spin-down power distribution,

- the uncertainties on their time evolution, in terms of the braking index $\kappa$ and characteristic spin-down timescale $\tau_{0}$,

- the fraction of spin-down power that goes to cosmic-ray electrons and positrons released into the interstellar medium, and the injection spectrum these particles have,

- how the electrons/positrons propagate from the pulsars to us i.e. their propagation through the ISM and the Heliosphere.

In this work we produce 7272 Milky Way pulsar simulations to account for the various combinations of parameters that we vary. Each of our simulations extends out to $4 \mathrm{kpc}$ from the Sun and contains between $5 \times 10^{3}$ to $19 \times 10^{3}$ unique pulsars, depending on the exact assumption of the Milky Way pulsar birth rate. In the following we describe the specific assumptions that we test in our simulations.

\section{A. The birth distribution of pulsars in space and time}

From observations along the galactic plane we expect a pulsar birth rate of $1.4 \pm 0.2$ per century [120]. However, this rate is probably more uncertain as wider estimates have been made in [117-119, 121]. We take three basic choices for the pulsar birth rate of $0.6,1$ and 2 per century 
for the entire Milky Way. We note that more choices for the birth rate between the values of 0.6 and 2 pulsars per century would not change our basic results ${ }^{2}$.

In our simulation pulsars are stochastically generated based on a profile probability density function. We follow the same spatial distribution as in earlier work of [122]. Our spatial distribution relies on observations of Parkes multi-beam pulsar survey at $1.4 \mathrm{GHz}$ [124] and subsequent models in [119, 120, 125]. We assume azimuthal symmetry with respect to the galactic center and an exponentially decreasing pulsar density as we move away from the galactic disk, with a typical scale height of 50 pc. As most of the rotational energy of pulsars is emitted in their first $O(10) \mathrm{kyr}$, the associated natal kicks that are typically $O\left(10^{2}\right) \mathrm{km} / \mathrm{s}$, can only result in a displacement of $O(1)$ pc. Such displacements represent a minor correction to the original birth spacial distribution which we ignore.

\section{B. The Pulsars' Initial Spin-Down Distribution Properties}

Each pulsar due to asymmetries in the core-collapse of their progenitor star acquires an initial rotational energy. At the same time these are highly magnetized objects with initial B-field strengths at their poles of order $10^{12}$ $\mathrm{G}$ and up to $10^{15} \mathrm{G}$ for magnetars. Typically the axis of rotation is not aligned to the axis of the magnetic field leading to energy losses, known as spin-down. A pulsar's spin-down power $\dot{E}$ evolves with time and is modeled here as [126],

$$
\dot{E}(t)=\dot{E}_{0}\left(1+\frac{t}{\tau_{0}}\right)^{-\frac{\kappa+1}{\kappa-1}} .
$$

$\dot{E}_{0}$ is the original spin-down power of a given pulsar, $\tau_{0}$ is its characteristic spin-down timescale and $\kappa$ its braking index. For a given Milky Way pulsars simulation we assume that the pulsars' initial spin-down power is equal to $\dot{E}_{0}=10^{x} \mathrm{ergs} / \mathrm{s}$ with $x=x_{\text {cutoff }}-y$. In each of our Milky Way simulations each pulsar has its unique $y$-value, i.e unique initial spin-down power. Using the same parametrization of [122], the $y$-parameter follows a log-normal distribution,

$$
f(y)=\frac{\operatorname{Exp}\left\{-\frac{\left[-\mu_{y}+\ln (y)\right]^{2}}{2 \sigma_{y}^{2}}\right\}}{\sqrt{2 \pi} y \sigma_{y}} .
$$

The values for $x_{\text {cutoff }}$ and $\mu_{y}$ are constrained by radio observations and subsequent modeling of Myr old pulsars' periods [119]; and by comparing to the ATNF pulsar catalog [127, 128]. We set an upper cutoff $x<x_{\max }=38.7$

\footnotetext{
2 Our simulations show a preference for a birth rate of 2 pulsars per century, but not in a manner that changes our conclusions.
}

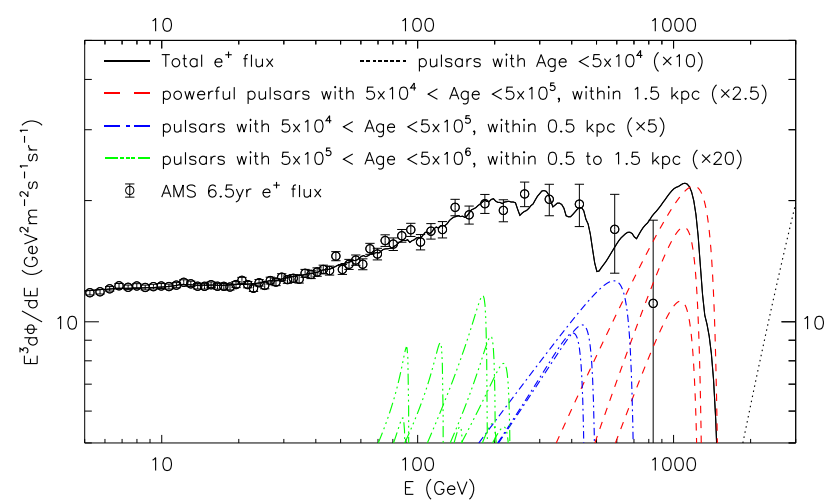

FIG. 1. The cosmic-ray positron flux form a Milky Way pulsars simulation. The solid black line includes the contribution of the cosmic-ray secondaries (from inelastic p-p, p-N and N$\mathrm{N}$ collisions in the ISM). We highlight the contribution to the positron flux from individual pulsars that have ages from $O(10)$ kyr to 5 Myr and that are relatively close-by. The contribution from individual pulsars is enhanced from the original simulation to show their fluxes within the figure. The $A M S$-02 observation is shown in the data points.

indicative of the Crab pulsar's observed spin-down . We take $\sigma_{y}=[0.25,0.36,0.5,0.75]$ which allow varying degree of widths in those distributions. A $\sigma_{y}=0$ would assume that all pulsars have an identical initial spin-down power. A distribution on the $y$-parameter should be expected both from the fact that there is a distribution on the magnitude of the initial B-fields at the poles and a distribution in the angle between the magnetic field axis and the axis of rotation.

\section{The Pulsars' Spin-Down Evolution}

As we said in the introduction we want to test the spin-down properties of pulsars relying on cosmic-ray observations. Detected cosmic-rays at the $5 \mathrm{GeV}-5 \mathrm{TeV}$ energy range can be used to probe the contribution of pulsars with ages between $O\left(10^{2}\right) \mathrm{kyr}$ and $O\left(10^{2}\right) \mathrm{Myr}$ as we show in Figure 1. where we have highlighted the contribution of a few specific pulsars of given distance and age ranges. Younger pulsars contribute at $\mathrm{TeV}$ energies. Middle-aged pulsars of age $10^{2}-10^{3} \mathrm{kyr}$ contribute dominantly at $O(100) \mathrm{GeV}$ and can give spectral features at these energies. Older pulsars have suppressed overall fluxes but also more prominent peaks and subsequent cut-offs, due to cosmic-ray cooling, and might still be able to give minor spectral features at energies lower than $100 \mathrm{GeV}$.

As we want to test if the braking index $\kappa$ is different for $O\left(10^{2}\right)-O\left(10^{5}\right)$ kyr pulsars compared to the much younger objects, we create Milky Way pulsars simulations where all pulsar members have the same value of $\kappa$ and $\tau_{0}$. By creating such simulations we can test if pulsars of older ages statistically prefer certain values of $\kappa$. 
If a pulsar's angle between its axis of rotation and magnetic field axis evolves with time, that can be interpreted as an evolution of $\kappa[129,130$. A relatively fast decay of the magnetic field's amplitude at the poles can also lead to a changing braking index 131-136. In addition, different equations of state can give for the same total mass different values of $\kappa$ [137, 138]. All these effects can result in the braking index evolving with time. Only a small number of pulsars exist with a reliably measured braking index, and all of them are very young [139, 140, (see however [141]). Such young pulsars have negligible contribution to the observed cosmic-ray spectra and are not the focus of this study.

In TableI, we give all the spin-down power distribution properties for our Milky Way pulsars simulations. We test discrete values of $\kappa=[2.5,2.75,3.0,3.25,3.5]$, where $\tau_{0}$ vary from $0.6 \mathrm{kyr}$ for $\kappa=2.5$ to $30 \mathrm{kyr}$ for $\kappa=3.5$. These combination of values of $\tau_{0}$ and $\kappa$ are picked to be in agreement with surface magnetic fields as well as periods from [119]. We also include different assumptions on the pulsars' distribution of the initial spin-down power. Table I is an expansion of earlier work in [122. We typically run 72 simulations per combination of $\tau_{0}, \kappa, x_{\text {cutoff }}$, $\mu_{y}$ and $\sigma_{y}$, but in some cases we run up to 108 (as in the 100-1H7) case.

\section{Pulsars as Sources of Cosmic-Ray Electrons and Positrons}

From microwave, X-ray and gamma-ray observations, we know that high-energy cosmic-ray electrons and positrons exist within a pulsar's magnetosphere. Moreover, electrons and positrons can be further accelerated in the termination shock of the pulsar wind nebula (PWN) and if there still is a supernova remnant shock (SNR), its termination shock just before entering the ISM. In addition observations from HAWC and Milagro show evidence for $O(10) \mathrm{TeV} \gamma$-rays towards Geminga and Monogem [30, 142, 143. These observations show that 10-100 $\mathrm{TeV}$ cosmic-ray electrons and positrons exist at distances up to $\simeq 10$ pc from these middle aged pulsars.

In Ref. 40, it was shown that as a pulsar's characteristic spin-down timescale $\tau_{0}$ is $\sim 10^{4} \mathrm{yrs}$, an appreciable fraction $(\sim 1 / 2)$ of the pulsar's initial rotational energy will be lost before the SNR shock front surrounding it stops being an efficient cosmic-ray accelerator. $\tau_{0}$ is also much smaller than the timescale the surrounding PWN stops accelerating cosmic-rays. As cosmic rays typically require $O\left(10^{2}\right)$ kyr to Myr to reach us from individual pulsars through diffusion, we can reliably approximate pulsars to instantaneously inject cosmic-ray electrons and positrons at the time of their birth (see Ref. [40] for further details) 3 .

\footnotetext{
${ }^{3}$ An exception to that approximation would be the injection of
}

The aim of this work is to constrain the averaged cosmic-ray injection spectral index $n$ from pulsars. For the injection spectra we assume,

$$
\frac{d N}{d E} \propto E^{-n} \operatorname{Exp}\left\{-\frac{E}{E_{\text {cut }}}\right\} .
$$

We take $n$ to follow a uniform distribution within the range of $n \in[1.4,1.9]$ which we refer to as option "A" or two narrower ranges of $n \in[1.6,1.7]$, our option "B", or $n \in[1.3,1.5]$ our option "C". The upper cutoff $E_{\text {cut }}$ is taken to be $10 \mathrm{TeV}$. We find that its exact value does not affect our fitting results. This should be expected as the highest energy cosmic rays lose their energy faster.

Furthermore, we model the fraction $\eta$ of rotational energy that ends in cosmic-rays released into the ISM and the relative variations of that fraction between pulsars. Following 69, 122, we take a log-normal distribution for the $\eta$ parameter,

$$
g(\eta)=\frac{\operatorname{Exp}\left\{-\frac{[-\mu+\ln (-1+\eta)]^{2}}{2 \sigma^{2}}\right\}}{\sqrt{2 \pi}(\eta-1) \sigma},
$$

and take three different choices for $(\mu, \sigma)$ to be $(0.32$, 0.12) (option "A"), (0.64,0.23) (option "B") and (-0.38, 0.16 ) (option "C"). These give square root variances of $0.169,0.454$ and 0.112 respectively. In our Milky Way pulsars simulations before fitting to the data we also pick specific values for $\mu$ that affect the mean efficiency of these pulsars, $\bar{\eta}=1+\operatorname{Exp}\left\{\mu+\frac{\sigma^{2}}{2}\right\}$. These are $\bar{\eta}=$ $4 \times 10^{-3}$ (for option "A"), $10^{-3}$ (for option "B") and $2 \times 10^{-2}$ (for option "C"). However, we fit each Milky Way pulsars simulation to the data and thus the $\bar{\eta}$ is reset by the data.

In Figure 2, we show five simulations of Milky Way pulsars to highlight the impact of our assumptions on the barking index $\kappa$, the spin-down timescale $\tau_{0}$ and the standard deviation of the fraction of energy going to cosmic-ray electrons and positrons variance, where $10^{\sqrt{\text { variance }}}=\zeta$. We simulate $8.6 \times 10^{3}$ individual pulsars, whose locations and ages are fixed. The youngest of these pulsars is $175 \mathrm{yr}$ and the oldest $10 \mathrm{Myr}$, and all are with $4 \mathrm{kpc}$ from us. We change the spin-down evolution of those pulsars by assuming different values of $\kappa$ and $\tau_{0}$ relevant in Eq. 2 taking a fixed value of $\zeta$ (red vs black vs blue solid lines). We normalize all simulations in Figure2 to get the same positron flux at $100 \mathrm{GeV}$. This is done to showcase the impact of these assumptions in our analysis. As we will describe in section III, we fit the simulated fluxes to the $A M S-02$ observed flux. Once fitting

cosmic-rays from a very young pulsar within $<100$ pc. Such pulsars are very rare however, appearing in very few of our simulations. Moreover, as we show in Figure 1 1 their contribution would appear at very high energies, not observable by the satellite experiments we rely on. 


\begin{tabular}{c|ccccc} 
Sim no. & $\tau_{0}(\mathrm{kyr})$ & $\kappa$ & $x_{\text {cutoff }}$ & $\mu_{y}$ & $\sigma_{y}$ \\
\hline \hline $100-1 \mathrm{H} 7$ & 6 & 3 & 38.8 & 0.25 & 0.5 \\
$200-2 \mathrm{H} 7$ & 3.3 & 3 & 38.8 & 0.25 & 0.5 \\
$300-3 \mathrm{H} 7$ & 10 & 3 & 38.8 & 0.25 & 0.5 \\
$400-471$ & 3.3 & 3 & 39 & 0.1 & 0.5 \\
$500-571$ & 1 & 2.5 & 38.8 & 0.25 & 0.5 \\
$600-671$ & 20 & 3.5 & 39 & 0.1 & 0.5 \\
$700-771$ & 0.7 & 2.5 & 38.8 & 0.25 & 0.5 \\
$800-871$ & 20 & 3.5 & 39.1 & 0.0 & 0.25 \\
$900-971$ & 0.6 & 2.5 & 39.0 & 0.1 & 0.25 \\
$1000-1071$ & 6 & 3 & 39.0 & 0.1 & 0.25 \\
$1100-1071$ & 6 & 3 & 38.7 & 0.5 & 0.75 \\
$1200-1271$ & 30 & 3.5 & 38.8 & 0.25 & 0.5 \\
$1300-1371$ & 0.85 & 2.5 & 38.5 & 0.6 & 0.75 \\
$1400-1471$ & 18 & 3.5 & 39.0 & 0.0 & 0.75 \\
$1500-1571$ & 10 & 3 & 38.7 & 0.5 & 0.75 \\
$1600-1671$ & 4 & 3 & 39.0 & 0.0 & 0.36 \\
$1700-1771$ & 1 & 2.5 & 38.7 & 0.5 & 0.75 \\
$1800-1871$ & 9 & 3 & 38.2 & 0.4 & 0.36 \\
$1900-1971$ & 0.8 & 2.5 & 38.2 & 0.4 & 0.36 \\
$2000-2071$ & 0.6 & 2.5 & 38.2 & 0.4 & 0.36 \\
$2100-2171$ & 30 & 3.5 & 38.2 & 0.4 & 0.36 \\
$2200-2271$ & 7 & 3 & 39.0 & 0.1 & 0.75 \\
$2300-23 \mathrm{H} 7$ & 30 & 3.5 & 38.0 & 0.5 & 0.36 \\
$2400-24 \mathrm{H} 7$ & 30 & 3.5 & 38.7 & 0.5 & 0.75 \\
$2500-25 \mathrm{H} 7$ & 6 & 3 & 38.9 & 0.18 & 0.36 \\
$2600-26 \mathrm{H} 7$ & 4.5 & 3 & 39.3 & 0.0 & 0.25 \\
$2700-27 \mathrm{H} 7$ & 9 & 3 & 38.5 & 0.5 & 0.25 \\
$2800-28 \mathrm{H} 7$ & 27 & 3.5 & 38.5 & 0.3 & 0.25 \\
$2900-29 \mathrm{H} 7$ & 33 & 3.5 & 38.0 & 0.5 & 0.25 \\
$3000-3071$ & 0.85 & 2.5 & 38.3 & 0.5 & 0.25 \\
$3100-3171$ & 18 & 3.25 & 38.8 & 0.25 & 0.5 \\
$3200-3271$ & 15 & 3.25 & 38.8 & 0.25 & 0.5 \\
$3300-3371$ & 18 & 3.25 & 38.5 & 0.4 & 0.25 \\
$3400-3471$ & 20 & 3.25 & 38.0 & 0.4 & 0.25 \\
$3500-3571$ & 15 & 3.25 & 38.5 & 0.4 & 0.36 \\
$3600-3671$ & 20 & 3.25 & 38.8 & 0.5 & 0.75 \\
$3700-3771$ & 2 & 2.75 & 38.8 & 0.25 & 0.5 \\
$3800-3871$ & 1.5 & 2.75 & 38.8 & 0.25 & 0.5 \\
$3900-3871$ & 1.6 & 2.75 & 38.5 & 0.5 & 0.25 \\
$4000-4071$ & 1.3 & 2.75 & 38.0 & 0.4 & 0.25 \\
$4100-4171$ & 1.2 & 2.75 & 38.3 & 0.4 & 0.36 \\
$4200-4271$ & 2.4 & 2.75 & 38.5 & 0.5 & 0.75
\end{tabular}

TABLE I. The Milky Way pulsars'-simulation spin-down power distribution and time evolution assumptions. We provide the names here as a reference to our publicly available list of simulation ${ }^{\mathrm{a}}$

a We have made publicly available our Milky Way pulsars simulations through,

https://zenodo.org/record/5659004\#. YYqnbi-ZNOs.

to the positron flux, a higher value of $\kappa$, would suggest that most flux from pulsars and the associated features are at higher energies. When pulsars have a larger $\kappa$ they release more slowly their energy. As a result they can remain strong sources of high-energy cosmic rays for a longer amount of time. In turn their combination gives enhanced fluxes at the highest energies.

In Figure 2, we also show the impact of varying the assumptions on $\sigma$, by fixing the spin-down evolution to $\kappa=3.0$ and $\tau_{0}=3.3 \mathrm{kyr}$. Larger values of $\sigma$ in Eq. 5 .

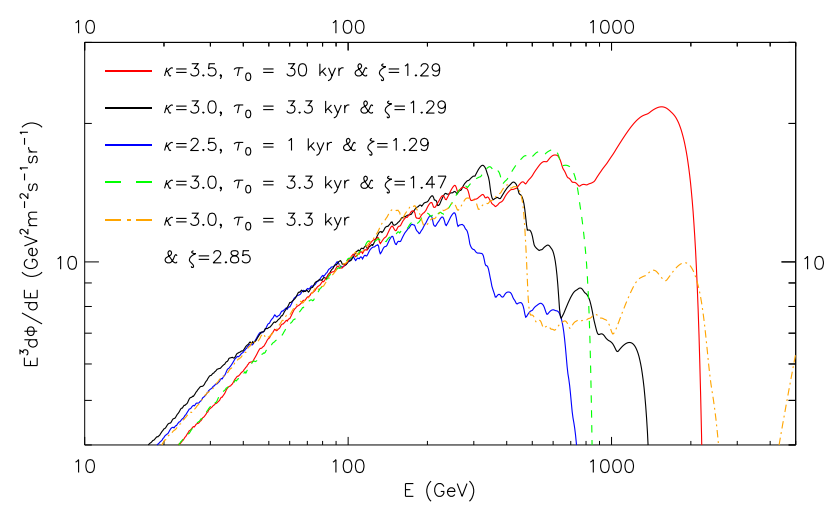

FIG. 2. The positron flux for different choices of spin-down evolution (red vs black vs blue) for a fixed assumption on $\zeta=10^{\sqrt{\text { variance }}}$. We also compare different choices for $\zeta$ by keeping the spin-down assumptions fixed (black solid vs green dashed vs orange dot-dashed). See text for details.

result in some pulsars depositing a great fraction of their energy in electrons and positrons. Such pulsars are responsible for prominent features in the positron flux (and the positron fraction). A similar effect exists if instead pulsars have a large variance in their initial spin-down power, i.e. the value of $\sigma_{y}$ of Eq. 3 is chosen to be high.

\section{E. Cosmic-Ray Propagation through the ISM and heliosphere}

Cosmic-ray electrons and positrons propagate in the interstellar medium via diffusion. How fast cosmic rays diffuse depends on the galactic magnetic field's amplitude and structure, and their energy. We assume isotropic and homogeneous diffusion that can be described by a rigidity $(R)$-dependent diffusion coefficient,

$$
D(R)=D_{0}\left(\frac{R}{1 G V}\right)^{\delta} .
$$

$D_{0}$ is the relevant normalization set at $1 \mathrm{GV}$, regulated by the overall strength of magnetic fields in the Milky Way, while the diffusion index $\delta$ is defined by the spectrum of interstellar turbulence. $\delta=0.33$, is for the case of Kolmogorov turbulence [144], while $\delta=0.5$ is for Kraichnan two-dimensional turbulence [145]. Systematic study of cosmic ray observations suggest that the diffusion index $\delta$ is within that range of values (see e.g. [48, 146]); which we use in our simulations. We model the diffusion taking place within a cylinder centered at the galactic center, of radius $20 \mathrm{kpc}$ and extending to a height of $\pm z_{L}$ away from the galactic disk (the disk is at $z=0$ ). Beyond those limits cosmic rays will escape the galaxy.

Furthermore, cosmic-ray electrons and positrons at the $10 \mathrm{GeV}$ to $10 \mathrm{TeV}$ range lose rapidly energy through inverse Compton scattering and synchrotron radiation with 


\begin{tabular}{ccccc}
\hline Model & $z_{L}(\mathrm{kpc})$ & $b\left(\times 10^{-6} \mathrm{GeV}^{-1} \mathrm{kyrs}^{-1}\right)$ & $D_{0}\left(\mathrm{pc}^{2} / \mathrm{kyr}\right)$ & $\delta$ \\
\hline \hline A1 & 5.7 & 5.05 & 140.2 & 0.33 \\
A2 & 5.7 & 8.02 & 140.2 & 0.33 \\
A3 & 5.7 & 2.97 & 140.2 & 0.33 \\
C1 & 5.5 & 5.05 & 92.1 & 0.40 \\
C2 & 5.5 & 8.02 & 92.1 & 0.40 \\
C3 & 5.5 & 2.97 & 92.1 & 0.40 \\
E1 & 6.0 & 5.05 & 51.3 & 0.50 \\
E2 & 6.0 & 8.02 & 51.3 & 0.50 \\
E3 & 6.0 & 2.97 & 51.3 & 0.50 \\
F1 & 3.0 & 5.05 & 33.7 & 0.43 \\
F2 & 3.0 & 8.02 & 33.7 & 0.43 \\
F3 & 3.0 & 2.97 & 33.7 & 0.43 \\
\hline
\end{tabular}

TABLE II. The ISM parameters that describe the propagations assumptions of cosmic rays in the Milky Way.

a rate that scales as,

$$
\frac{d E}{d t}=-b\left(\frac{E}{1 G e V}\right)^{2}
$$

This makes the highest energy cosmic-ray electrons and positrons lose more rapidly their energy and causes a "pile-up" before the cut-off, in the electron/positron fluxes from individual pulsars that is seen in Figure 1. The value of $b$, set at $1 \mathrm{GeV}$, is directly proportional to the energy density in the galactic magnetic field and the energy density in the CMB and interstellar radiation field photons. We note that at the highest energies the inverse Compton cross-section is not the Thomson crosssection assumed in Eq. 7, but instead the Klein-Nishina one [147, 148]. In our pulsars simulations we ignore the Klein-Nishina corrections, as we use a wide range of uncertainty on the $b$ parameter, set to be within $3 \times 10^{-6}$ and $8 \times 10^{-6} \mathrm{GeV}^{-1} \mathrm{kyrs}^{-1}$. We also ignore Bremsstrahlung emission losses that cause an energy-loss rate $\propto E$, that become important only at $\mathrm{GeV}$ energies.

Cosmic rays also experience diffusive reacceleration [149 and are affected by local convective winds. To account for ISM diffusion uncertainties we use four distinctive models, defined by the letters A, C, E, F. These models are in agreement with $A M S$-02 observations of the cosmic-ray proton, helium, carbon, oxygen fluxes and the beryllium-to-carbon, boron-to-carbon and oxygen-tocarbon ratios 49. Each one of these models has three variants to account for uncertainties in the energy losses, i.e the $b$-parameter, denoted by a second character (13 ). A value of $b=5.05 \times 10^{-6} \mathrm{GeV}^{-1} \mathrm{kyrs}^{-1}$ (for models A1, C1, E1, and F1), is in agreement with evaluations of the local magnetic and interstellar radiation field [43, 150, 151, while the choices of $2.97 \times 10^{-6}$ and $8.02 \times 10^{-6} \mathrm{GeV}^{-1} \mathrm{kyrs}^{-1}$ represent the relevant uncertainties. All these parameters are described in Table II.

In comparing the ISM model predictions to the $A M S$ 02 cosmic-ray nuclei measurements we used GALPROP v54 [43, 150] where we have included convection, reacceleration and Bremsstrahlung energy-losses. GALPROP gives us a prediction for the primary cosmic-ray electrons (from

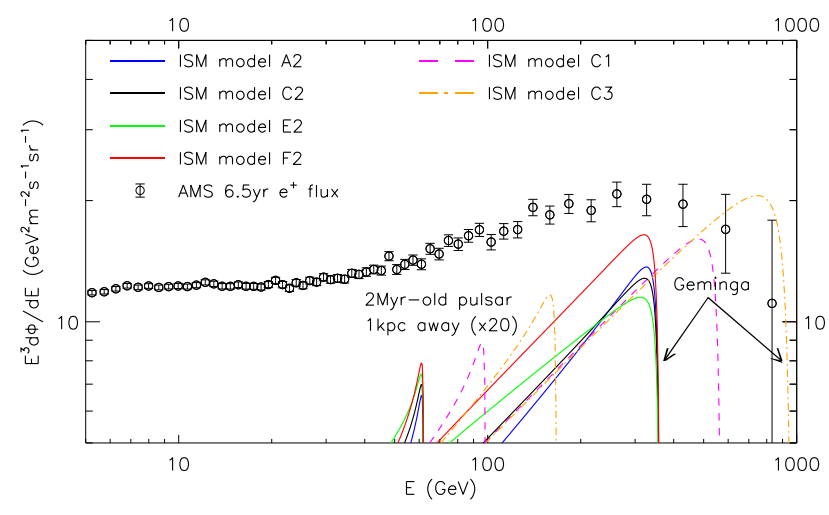

FIG. 3. The impact of different ISM assumptions on the positron flux from two individual pulsars. Solid lines (blue, black, green and red) show the impact of different diffusion assumptions. The magenta dashed and orange dot-dashed are to be compared to the black solid line that is made under the same diffusion assumptions.

SNRs) and the secondary electrons and positrons from $p-p, p-N$ and $N-N$ inelastic collisions taking place at the ISM gas. However, for the cosmic ray electrons and positrons from the individual pulsars the code that we use ignores ISM convection, diffusive reacceleration and Bremsstrahlung energy losses [40, 69, 122. In the energies of interest the timescale for these effects are significantly larger than the diffusion and energy losses of Eq. 7 timescales. This allows us to place within $4 \mathrm{kpc}$ from the location of the Sun up to $1.9 \times 10^{4}$ pulsars in unique positions and of unique age created in the last 10 Myr. We take the primary electron fluxes and secondary electron and positron fluxes from GALPROP and combine them with the pulsars' electron and positron fluxes from our Milky Way pulsars simulations.

In Figure 3, we show how the positron flux from individual pulsars is affected by different ISM propagation conditions. We model two individual pulsars, Geminga that is taken to be $0.25 \mathrm{kpc}$ away and $3.42 \times 10^{5} \mathrm{yr}$ in agreement with observations [128, and a second pulsar $1.0 \mathrm{kpc}$ away and $2.0 \times 10^{6} \mathrm{yr}$ old. Both pulsars are taken to have an initial spin-down power of $\dot{E}_{0}=1.1 \times 10^{38}$ $\mathrm{erg} / \mathrm{s}$, braking index $\kappa=3.0$ and $\tau_{0}=6.0 \mathrm{kyr}$, that for Geminga would give the currently observed spin-down power of $3.2 \times 10^{34} \mathrm{erg} / \mathrm{s}$. The cosmic-ray injection index of Eq. 4 is taken to be $n=1.65$ for both.

Keeping energy losses fixed and changing between A2, $\mathrm{C} 2$ and E2 we note the difference in the positron flux's power-law spectrum for energies lower than the sharp cooling cut-off. That is simply the affected by the diffusion index $\delta$. For model F2 the flux is significantly larger as the diffusion normalization $D_{0}$ is the smallest to account for its small scale height of $z_{L}=3 \mathrm{kpc}$. Cosmicray electrons/positrons from close-by pulsars stay longer close of their source for smaller values of $D_{0}$. That is most evident in Figure 3 with Geminga. The energy losses set the value of the cooling cut-off. 
The observed cosmic-ray spectra by $A M S$-02 are affected by solar modulation. Cosmic rays have to travel through the Heliosphere before being recorded. During their propagation through the Heliosphere, cosmic rays diffuse through a fast evolving anisotropic magnetic field, experience drift effects and adiabatic losses. As a result their energy gets statistically shifted to lower values, described by the solar modulation potential $\Phi$ [152]. We use here the time-, charge- and rigidity-dependent formula for the solar modulation potential from [153], that has recently been further constrained in 154. Our model for solar modulation requires as inputs the value of the total B-field of the Solar Wind at $1 \mathrm{AU}$, which we take from $A C E$ [155, the tilt angle of the heliospheric current sheet that is modeled in Wilcox Solar Observatory 156. and the polarity of the Heliospheric magnetic field. In turn it gives us a value for the solar modulation potential that the kinetic energy of a particle of mass $m$, rigidity $R$ and charge $q$ was shifted by at a given Bartels' Rotation number. Our solar modulation model has two freeparameters $\phi_{0}$ and $\phi_{1}$ that we marginalize over within a range of values most recently constrained in [154]. For further details see [153, 154].

\section{COSMIC RAY DATA AND FITTING PROCEDURE}

In this section we describe the observational data that we use to test our pulsars population models and the specifics of the fitting procedure that we follow. We account for uncertainties in the overall normalization of the pulsars' contribution, and for spectral and normalization uncertainties of other components in the electron and positron cosmic-ray spectra.

\section{A. Observations of cosmic-ray electrons and positrons}

We use the publicly available $A M S$-02 positron flux, the positron fraction and the $e^{+}+e^{-}$flux from [55, 56, 157. Specifically for the $e^{+}+e^{-}$flux we rely on [56] instead of [55]. The analysis of [56] avoids charge sign identification and therefore results in a higher efficiency. For the $e^{+}+e^{-}$flux measurements we start at $10 \mathrm{GeV}$ to avoid possible low energy systematics that may exist in comparing the $A M S$-02 measurement to that of DAMPE [158] or CALET [159]. For the positron flux and the positron fraction, we used the measurements at energies of $5 \mathrm{GeV}$ or higher. At lower energies the pulsars' contribution is expected to be subdominant and mostly affected by pulsars several kpc away that we do not model. Moreover, the lower-energy $e^{ \pm}$spectra are strongly affected by solar modulation that we account for, but also other propagation uncertainties as those of diffusive reacceleration and cosmic-ray convection in the ISM that we set to be present but do not further marginalize over.

\begin{tabular}{c||c|c|c}
\hline Dataset & Acquisition Era & BR \# & Ref. \\
\hline \hline$A M S-02 e^{+}$ & $5 / 2011-11 / 2017$ & $2426-2514$ & 157 \\
AMS-02 $e^{+} /\left(e^{-}+e^{+}\right)$ & $5 / 2011-11 / 2017$ & $2426-2514$ & {$[55$} \\
AMS-02 $e^{-}+e^{+}$ & $5 / 2011-11 / 2017$ & $2426-2514$ & {$[56$} \\
DAMPE $e^{-}+e^{+}$ & $12 / 2015-6 / 2017$ & $2488-2508$ & 158 \\
CALET $e^{-}+e^{+}$ & $10 / 2015-11 / 2017$ & $2486-2515$ & 159 \\
\hline \hline
\end{tabular}

TABLE III. The cosmic-ray measurements from AMS-02, $D A M P E$ and $C A L E T$ used in this analysis.

In addition, we perform fits ignoring the measurements below $15 \mathrm{GeV}$. At $\sim 12 \mathrm{GeV}$ there is a bump at the positron fraction that our simulations find it difficultly to fit. We will come back to the matter of possible interpretations to this bump in section V. Finally, we use the $e^{+}+e^{-}$flux measurements from DAMPE [158] and $C A L E T$ 159. The used datasets with their respective data acquisition era and the corresponding Bartels' Rotation (BR) Numbers are presented in Table III.

\section{B. Fitting}

We allow for up to seven free parameters to be optimized in our simulations. Two solar modulation parameters $\phi_{0}$ and $\phi_{1}$, three normalization factors $a, b$ and $c$ for the primary cosmic-ray $e^{-}$flux, secondary cosmic-ray $e^{ \pm}$fluxes and total pulsar $e^{ \pm}$fluxes, and two spectral indices $d_{1}$ and $d_{2}$ responsible for hardening or softening the primary $e^{-}$and secondary $e^{ \pm}$spectra by multiplying them with $(E / 1 \mathrm{GeV})^{d_{1}}$ and $(E / 100 \mathrm{GeV})^{d_{2}}$ respectively. We remind to the reader that the primary and secondary fluxes are already modeled to include specific energy losses, diffusion, diffusive re-acceleration, convection and ISM gas distribution assumptions. They also originate from a distribution model for all primary cosmic-ray sources. The additional normalizations $(a, b)$ and spectral freedoms $\left(d_{1}, d_{2}\right)$ are to account for uncertainties in the overall efficiency and number of the primary cosmicray sources, the total ISM gas density, the exact injection spectra of primary $e^{-}$spectra and cosmic-ray nuclei spectra that through their collisions give the secondary $e^{ \pm}$

We fit each produced simulation to each dataset via a $\chi^{2}$ minimization. While the AMS-02 measurements were acquired during the same era, we do not fit all the $A M S$-02 datasets simultaneously as they originate using different type of analysis cuts. Similarly, we do not try to fit simultaneously all $e^{-}+e^{+}$measurements from the three different experiments, as some are in statistical tension with each other.

We first fit each simulation to the $A M S$-02 positron flux. Then we focus on the realizations that can fit to the positron flux data within $2 \sigma, 3 \sigma$ or $5 \sigma$ from an expectation of $\chi^{2}$ of 1 for each degree of freedom (d.o.f.). For our fits of the the positron spectrum above $5 \mathrm{GeV}$ there are 59 data points being fitted with five parame- 
ters. Thus the $3 \sigma$ and $5 \sigma$ limits that we use translate to a $\chi^{2} /$ d.o.f. of 1.337 and 1.683 respectively. Instead, for the positron spectrum in energies $E \geq 15 \mathrm{GeV}$ there are 44 data points. The relevant $2 \sigma$ and $3 \sigma$ limits we present translate to $\chi^{2} /$ d.o.f. of 1.290 and 1.467 . In those fits we take $\phi_{0}$ and $\phi_{1}$ to be within $[0.1,0.6] \mathrm{GV}$ and $[0,2] \mathrm{GV}$ respectively. Our range for the normalization $b$ is $[0.8,2]$. The parameter $c$ is only given an upper bound such that $\bar{\eta} c \leq 0.5$ for each realization, while the parameter $d_{2}$ is within $[-0.1,0.1]$.

We repeat the same fitting procedure with the $A M S-02$ positron fraction and $e^{-}+e^{+}$flux; where for those we make use of all seven free parameters. We focus only on the simulations that are within the $2 \sigma, 3 \sigma$ or $5 \sigma$ positron flux limits. For each of those simulations we take the best fit values of $b, c$ that we got from the relevant positron flux fit and allow for up to a $50 \%$ additional variation 4 . Parameters $\phi_{0}, \phi_{1}$ are taken within the same ranges reported above as we consider these to be quite wide, while $d_{2}$ is fixed at its best fit value from the positron flux. Finally, the two newly introduced parameters $a$ and $d_{1}$ relating to the primary $e^{-}$component take values within the range of $[0.6,1.2]$ and $[-0.2,0.5]$ respectively.

We report the simulations that can fit each of the $A M S$ 02 data, i) within $2 \sigma$ and $3 \sigma$ for energies of $E>15$ and ii) within $3 \sigma$ and $5 \sigma$ for $E \geq 5 \mathrm{GeV}$ for the positron flux and fraction and $E>10$ for the total $e^{-}+e^{+}$flux. We then compare the retained simulations from our $E>15$ fits to the DAMPE and CALET data. The DAMPE and $C A L E T$ fits are performed in the same manner as the $A M S-02 e^{+}+e^{-}$flux.

When we fit the positron fraction in each energy bin we use counts instead of fluxes as $A M S$-02 uses binned data. At the highest energies some of our simulations may suggest the presence of multiple features within the same energy bin. Thus, we integrate the differential flux $d \Phi^{e^{ \pm}} / d E$ over the energy range of each bin, and set a number of positron and electron events $N^{e^{ \pm}}$as,

$$
N^{e^{ \pm}}=\int_{E_{\min }}^{E_{\max }} \frac{d \Phi^{e^{ \pm}}}{d E} d E
$$

where $E_{\min }, E_{\max }$ are the bounds of the bin. We note that $N^{e^{ \pm}}$is not the real event count in each bin as we don't know the exact exposure of the $A M S$-02 detector. For any given energy bin assuming the exposure is roughly constant, ignoring it, is not an issue as the exposure cancels in taking the positron fraction ratio.

The positron fraction becomes,

$$
\frac{b N_{e^{+}}^{s e c}+c N_{e^{+}}^{p u l}}{a N_{e^{-}}^{p r i}+b N_{e^{-}}^{s e c}+c N_{e^{-}}^{p u l}+b N_{e^{+}}^{s e c}+c N_{e^{+}}^{p u l}} .
$$

\footnotetext{
4 We always retain the hard limit of $\bar{\eta} c \leq 0.5$ that originates from equipartition of spin-down power to cosmic rays and B-field.
}

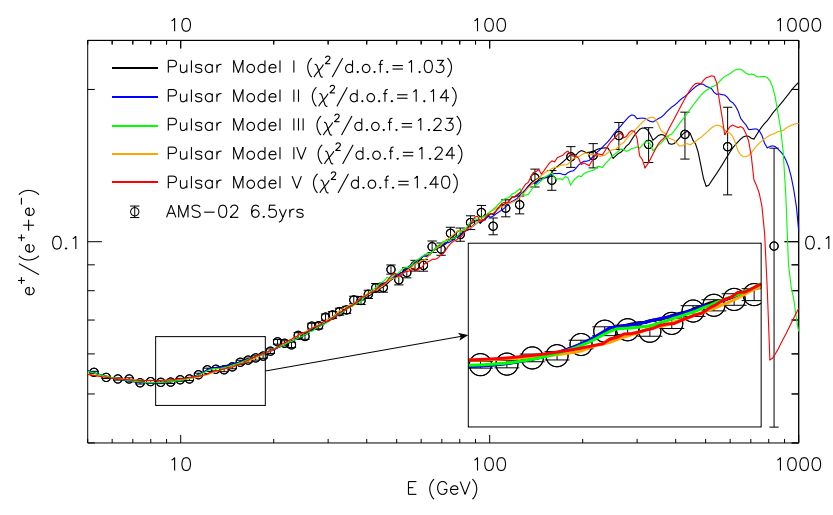

FIG. 4. The predicted positron fraction spectra for five different pulsar realizations along with the AMS-02 data. The $\chi^{2} /$ d.o.f. of these pulsar models are also shown, evaluated at energies greater than $5 \mathrm{GeV}$. The zoomed part shows the spectrum around the bump that is centered at $\simeq 12 \mathrm{GeV}$.

The indices "pri", "sec", "pul" refer to the primary, secondary and pulsar fluxes respectively. We note that the factors $(E / 1 \mathrm{GeV})^{d_{1}}$ and $(E / 100 \mathrm{GeV})^{d_{2}}$ have been absorbed into their respective counts. For the positron flux or the $e^{+}+e^{-}$flux fits using "counts" is not possible as we do not know the exact exposures. Therefore we remain on working with fluxes.

Our minimization procedure uses SciPy's [160] least_ squares routine from the optimize module to solve our non-linear least squares problem. We also tried iMinuit 161 but found out that the least_squares achieves a good minimization much faster 5

\section{RESULTS}

The first step in testing every Milky Way pulsars simulation is to fit its predicted positron flux in combination with a secondary positron flux component to the AMS-02 positron flux measurement, as shown for one model in Figure 1. The second step is to test that simulation against the positron fraction measurement and the $e^{+}+e^{-}$measurement (see Section III B for details). Our secondary and primary flux components are evaluated for the same diffusion and energy losses assumptions as the electrons and positrons fluxes originating from pulsars.

In Figure 4, we show the positron fraction spectra from different pulsars simulation assumptions. There is a clear

\footnotetext{
${ }^{5}$ We noticed that the starting value for the parameter $d_{1}$ can cause the minimization in the positron fraction to fall into local minima. Therefore we minimize each simulation several times, starting from different values for $d_{1}$ in its allowed range. In each minimization all the other parameters' starting values are chosen randomly within their allowed ranges as we found that their exact starting value doesn't cause any issues.
} 


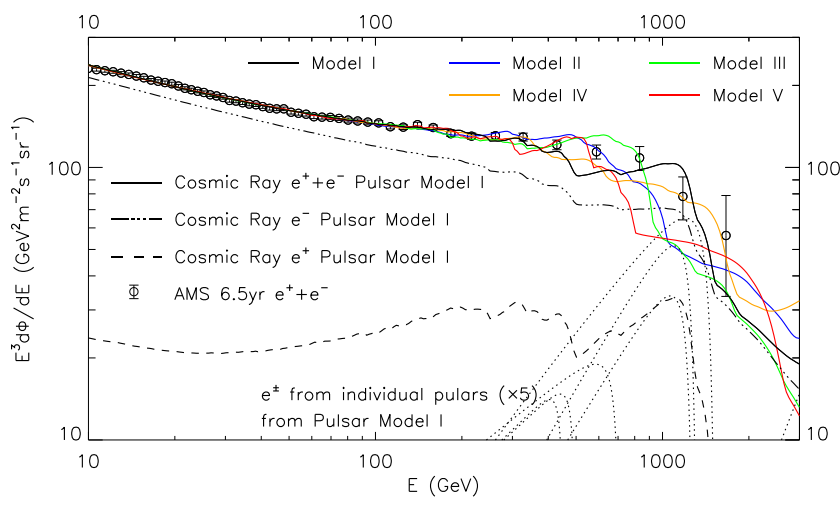

FIG. 5. The predicted $e^{+}+e^{-}$spectrum for the same five pulsar simulations of Figure 4 along with the AMS-02 observation. For model I, we also show the individual electron and positron spectra and fluxes from individual pulsars.

feature that peaks around $12 \mathrm{GeV}$. A similar feature exists also in the positron flux. In presenting our results we break our discussion into two subsections. The first one IV A is focused on fitting only energies above 15 $\mathrm{GeV}$ avoiding the impact of a such a feature. Instead in Section IVB, we include the energies of 5 to $15 \mathrm{GeV}$ in our fits.

Pulsar model I of Figure 4, represents the best fit in the positron fraction from energies $5 \mathrm{GeV}$ and above. This same model gives a good fit at $E>15 \mathrm{GeV}$ as well. Our simulations include enough physical variations that the predicted positron fraction spectrum, which increases from 7 to $\sim 300 \mathrm{GeV}$ can either keep rising at higher energies (model I), drop (model II,III,V) or flatten out (model IV). We also note that our allowed pulsars simulations can have a noticeable amount of features in them, something that can be searched for independently (see [122] for a detailed discussion).

In Figure 5, we depict the five $e^{+}+e^{-}$spectra for the same pulsar models (I through V). For model I we plot the $e^{-}$and $e^{+}$fluxes separately and the fluxes from some individual pulsars. These simulations can fit the AMS-02 positron and the $e^{+}+e^{-}$fluxes well and have $\chi^{2} /$ d.o.f. $<1$, with the exception of model $\mathrm{V}$ that has $\chi^{2} /$ d.o.f. $=1.51$ in its $e^{+}+e^{-}$fit. Similarly to what was shown in Figure 1 for the positrons, individual pulsars can give features in the higher energies of the electron spectrum and possibly explain features in the combined $e^{+}+e^{-}$spectrum.

\section{A. Results for $E>15 \mathrm{GeV}$ fits}

\section{Using only AMS-02 data}

Of the 7272 astrophysical realizations, 2105 can fit the AMS-02 positron spectrum within $3 \sigma$ from an expectation of $\chi^{2}$ of 1 for each degree of freedom. Of these 2105

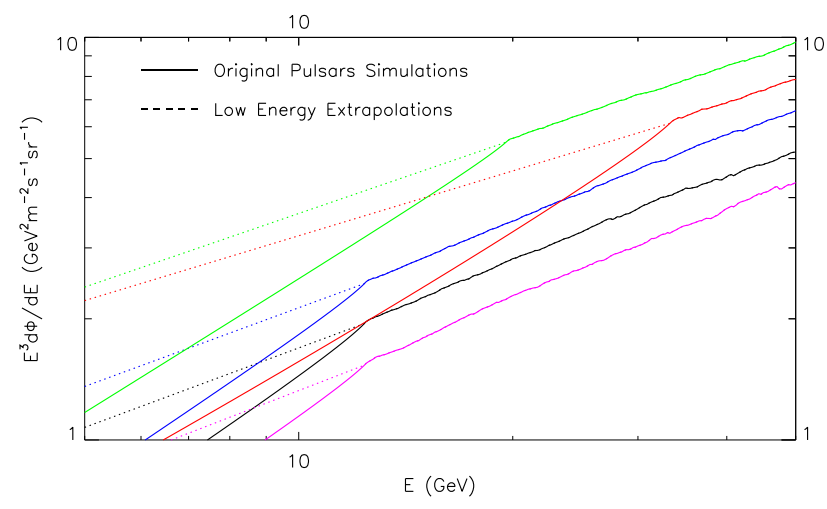

FIG. 6. Examples of low energy extrapolations on the $e^{ \pm}$flux from a sample of Milky Way pulsars simulations. Normalizations are arbitrary and fluxes do not include the impact of solar modulation. For each original simulation (solid lines) we evaluate the power law before the break and then extrapolate to lower energies (dotted lines).

realizations, 567 (1095) can also fit within $2 \sigma(3 \sigma)$ the $A M S-02$ positron fraction spectrum and $e^{+}+e^{-}$spectrum.

For every Milky Way pulsars simulation we perform a low energy extrapolation. We show in Figure 6, examples of that. This extrapolation accounts for the fact that our simulations end at $4 \mathrm{kpc}$ in distance from the Sun and at $10 \mathrm{Myr}$ in age of pulsars. Through this extrapolation, more distant and older pulsars that would contribute at lower energies are included. The total number of Milky Way pulsars simulations that we fit and compare to the observations is thus 14544 (instead of just 7272 simulations). However, we note that simulations that can provide a good fit to the data both with and without their low-energy extrapolation, count only once in the list of simulations that are in agreement with the data.

The exact ISM conditions beyond $4 \mathrm{kpc}$ are not simulated for the pulsars' electron/positron flux components. As the ISM properties gradually change we expect that there is increased modeling uncertainly at the lower energies and both options should be considered viable. Moreover, at low energies we include in our fits the impact of solar modulation with its uncertainties. This can further modify the fluxes' spectral properties at these low energies. We note that while lower birth rates result in small features at the lower energies, this does not affect our ability to perform that extrapolation. That can be seen in Figure 6, by comparing the blue vs black vs magenta lines that are for similar simulation assumptions, but with 2 vs 1 vs 0.6 pulsars/century birth rates respectively. The exact point where the low-energy extrapolation starts depends on the energy losses assumption. Lower energy losses (as the red line in Figure 6) result in a higher energy from which the extrapolation starts.

Our findings on the pulsar properties and the ISM conditions can be summarized in Figures 7,8 and 9 . We show in each cell the percentage of the pulsar population 


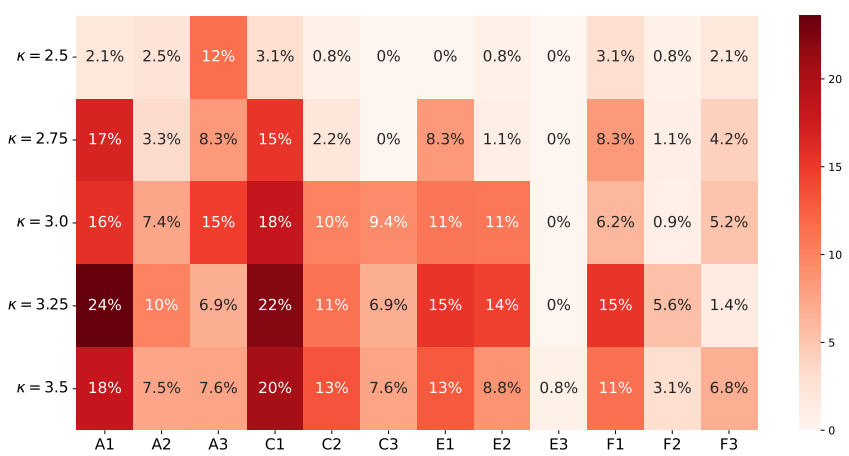

FIG. 7. In each cell we show the fraction of pulsar population simulations that are consistent within $2 \sigma$ to the $A M S-02$ positron fraction spectrum, the positron flux and the electron plus positron flux (see text for details) for the five choices of braking index $\kappa=2.5,2.75,3.0,3.25,3.5$ and the twelve choices of ISM propagation conditions modeled by "A1" to "F3" (see Table II).

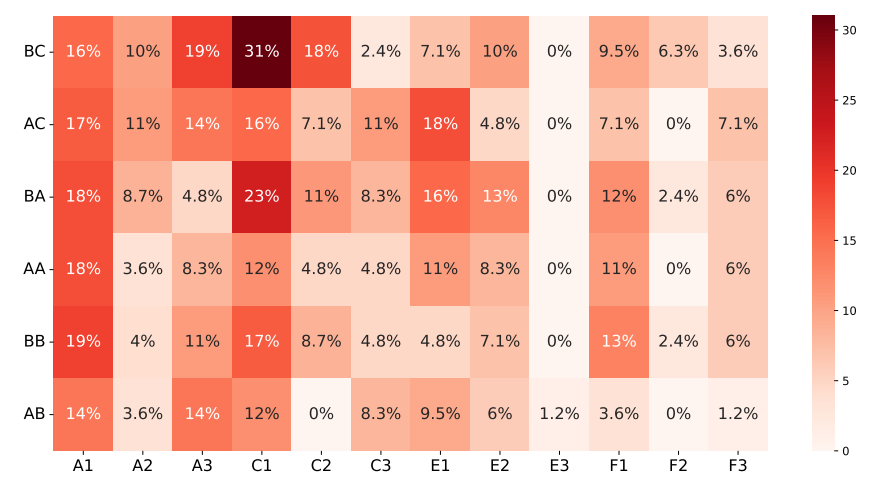

FIG. 8. Same as Figure 7 for the combination of the six choices ("BC", "AC", "BA", "AA", "BB" and "AB") of $n$ and $g(\eta)$ and the twelve ISM models.

simulations that are consistent within $2 \sigma$ to the AMS-02 positron fraction spectrum, the positron flux and electron plus positron flux. For instance, the top left cell of Figure 7 the value of 2.1 refers to the percentage among the simulations with $\kappa=2.5$, ISM model A1 assumptions, that are consistent with the $A M S$-02 data within $2 \sigma$.

In Figure 7, we show our results for the combination of the five choices of the braking index and the twelve choices of ISM propagation conditions. The choices of braking index $\kappa=2.5$ and $\kappa=2.75$ are clearly disfavored. Only a few realizations with these choices survive within $2 \sigma$. For $\kappa=3.0$ and even more so for $\kappa=3.25$ there is a significant increase on the fraction of simulations consistent to the data. Simulations with $\kappa=3.5$ are favored as much as simulations with $\kappa=3.0$. The preference for $\kappa \geq 3.0$ can also be seen in Figure 9. In Ref. [69], a first indication for a $\kappa \geq 3.0$ tendency was found. We now confirm this tendency with a much larger set of simulations, that account for even greater range of the relevant parameter space being modeled. For the

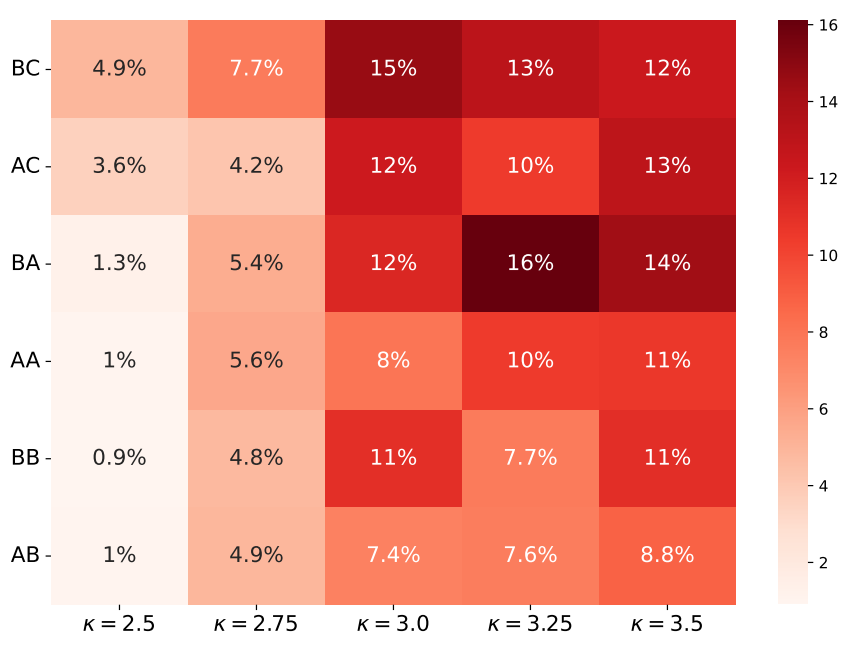

FIG. 9. Similar to Figures 7 and 8 , for the combination of the six choices of $n$ and $g(\eta)$ and the five choices for braking index $\kappa=2.5,2.75,3.0,3.25,3.5$.

$\sim 10$ young pulsars that we have reliable measurements of their breaking index, typical values are $\kappa<3$ [139, 140]. A higher value than 3.0 for the braking index, represents a slower spin-down (see Eq. 22 for middle aged and multiMyr old pulsars. While the sample of young pulsars is still small, one solution between the results from electromagnetic observations and the results of this analysis is that pulsars may increase their braking as they age. As we rely on observations from radio waves and evolve the pulsars back in time to calculate their total power, a constant braking index of $\kappa<3$, produces pulsars that initially were very powerful sources emitting very large amounts of cosmic-ray electrons and positrons. This increases the emission from the older pulsars compared to the younger ones.

As can be seen from both Figures 7 and 8 there is a small preference for the propagation models "A1, "C1", "E1" and "F1" which are for the more commonly used assumptions on the local cosmic-ray electrons and positron losses . Interestingly, practically $0 \%$ of our astrophysical realizations that have propagation model "E3" are consistent to the data. The "E3" simulations model low-energy losses and fast diffusion of high-energy cosmic rays. Also, models for a thin diffusion disk ("F1", "F2" and "F3") are disfavored. Thin disk ISM models make the cosmicray positrons even at low energies quite local and suppress the overall pulsars' contribution, making the combined spectrum from pulsars harder, something that is in tension to the observations.

In Figure 8, we present the results for the combination of the six choices for the injection index $n$ and the energy conversion to cosmic-rays $g(\eta)$ ("BC", "AC", "BA", "AA", "BB" and "AB") and the twelve ISM models. We clarify that there are three more choices ("CA", "CB" and "CC") for $n \in[1.3,1.5]$ that are not shown here. These would be redundant rows in the table as none of the realizations with these choices are within our $2 \sigma$ limit. The preferences on the ISM properties are there as we 
noted before. Regarding the index $n$ described by the first letter ("A" or "B") along the rows, there a slight preference for the choice " $\mathrm{B}$ " which is for a narrow range of values for $n \in[1.6,1.7]$ over the choice " $\mathrm{A}$ ", which is for a wider range of $n \in[1.4,1.9]$ (see Section IID for further details). The broader range for $n$ (under choice "A") results in a more diverse pulsar population with respect to their injected cosmic-ray electrons and positrons. Therefore pulsars under assumption "A" have quite diverse spectra. The resulting combined pulsars' spectrum in turn has some very pronounced features associated with pulsars that have $n$ closer to 1.4. Such a choice while not fully excluded is less preferable. Choice "C" which is fully excluded, assumed that all pulsars have values of $n \in[1.3,1.5]$. This resulted in spectra with too many strong spectral features compared to what is observed in the $A M S-02$ data. Our results suggest that pulsars in the Milky Way most likely have a small range of values for their spectral index $n$ with values of $n \simeq 1.6$.

In Figure 8, when comparing our results with respect to the choices of $g(\eta)$ (depicted by the second letter along the rows), we see a gradient going from our choice " $\mathrm{C}$ ", to choice "A" and then to "B". Those are ranked from smaller to larger variance on the fraction of power $\eta$ that goes to cosmic rays (see Section IID). Our results suggest that pulsars simulations with a greater homogeneity in the total spin-down power converted to comic-rays are preferred. If pulsars had a very large range of $\eta$, then even among the older pulsars contributing at the lower energies, we would have a few of them standing out in their produced fluxes. Those pulsars would again give more spectral features than what is observed.

In Figure 9, we present our results for the combination of the six choices for $n$ and $g(\eta)$, and the five choices for braking index. This figure, is given as a projection along that part of the simulations parameter space and very clearly shows the preferences for both $\kappa \geq 3.0$ and the choice "B", to the "A" for $n$.

\section{Observations at TeV energies from DAMPE and CALET}

The positron flux is measured by $A M S-02$ up to energies of $1 \mathrm{TeV}$. Two more experiments, DAMPE 162 and $C A L E T$ 163 have published their measurements of the total $e^{+}+e^{-} \mathrm{CR}$ flux up to $5 \mathrm{TeV}$ [158, 159. At these energies the pulsars' contribution to the total measured fluxes can be very significant. The combination of volume and age necessary for pulsars to be able to contribute, is reduced at these higher energies. We can only probe the properties of the youngest and most energetic pulsars that are also close-by members of the population. Those are small in number. The result is an $e^{+}+e^{-}$flux rich in spectral features. Such spectra can be seen in Figures 10 and 11 where we show the predicted $e^{+}+e^{-}$ fluxes for some of our pulsars models. Above $1 \mathrm{TeV}$, the spectra can either have a cut-off, a change in their slope,

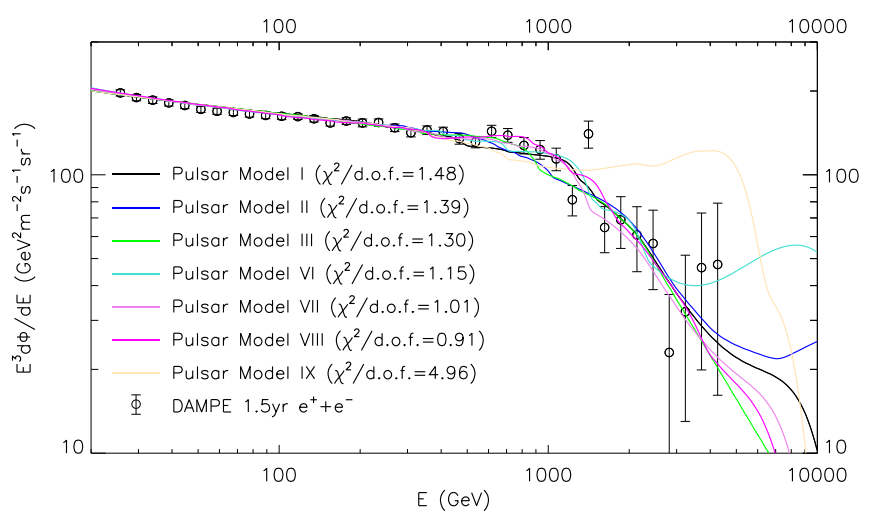

FIG. 10. The predicted $e^{+}+e^{-}$spectra for seven different pulsar models along with their $\chi^{2} /$ d.o.f. One of the models (model IX in beige color) is excluded by the DAMPE data. This model greatly overshoots the data points above $1 \mathrm{TeV}$. Some fluxes (such as model VI and VIII) contain notable features at $\mathrm{TeV}$ energies from individual powerful pulsars.

or in some cases one or more prominent bumps from individual pulsars.

Fitting our simulations to the DAMPE $e^{+}+e^{-}$spectrum, we can further constrain the local Milky Way pulsars properties. Of the 567 (1095) realizations that were within $2 \sigma(3 \sigma)$ to all three $A M S$-02 datasets, 268 (771) are also consistent within $2 \sigma(3 \sigma)$ to the DAMPE $e^{+}+e^{-}$ data. DAMPE allows roughly three quarters of our models at the $3 \sigma$ level and roughly half of our models at the $2 \sigma$ level. We note that DAMPE excludes models in a uniform manner from the heat maps of Figures 7,8 and 9. Thus our conclusions on the averaged properties of pulsars and the local ISM do not change. One difference is that with $D A M P E$ we find a small preference in retaining simulations with lower birth rates suggestive of the fact that smaller rates more easily produce spectral features as the ones seen in the DAMPE data. However, realizations that contain extremely powerful young and near-by pulsars can cause the $e^{+}+e^{-}$flux to overshoot the DAMPE data at the highest energies and are excluded. In Figure 10, we show the predicted $e^{+}+e^{-}$ flux from six pulsar models that are consistent with the $D A M P E$. We also show the flux from one model (Pulsar Model IX) that is not consistent with the DAMPE data exactly due to the presence of very powerful pulsars at energies that $A M S-02$ can not measure.

$C A L E T$ 's observations can not further constrain our astrophysical realizations. Of the 567 (1095) realizations that were consistent with all $A M S-02$ data at $2 \sigma$ $(3 \sigma)$, only one is excluded by the CALET $e^{+}+e^{-}$flux. $C A L E T$ has larger error bars compared to DAMPE. Almost all of our 1095 realizations that are within $3 \sigma$ to the $A M S$-02 measurements, end up with $\chi^{2} /$ d.o.f. $<1$ fit to the CALET data. In Figure 11, we show seven different $e^{+}+e^{-}$fluxes from realizations that fit the $C A L E T$ observation. Even our model IX, that was excluded by $D A M P E$ with $\chi^{2} /$ d.o.f. $=4.96$, can fit the $C A L E T$ data with $\chi^{2} /$ d.o.f. $=0.53$. 


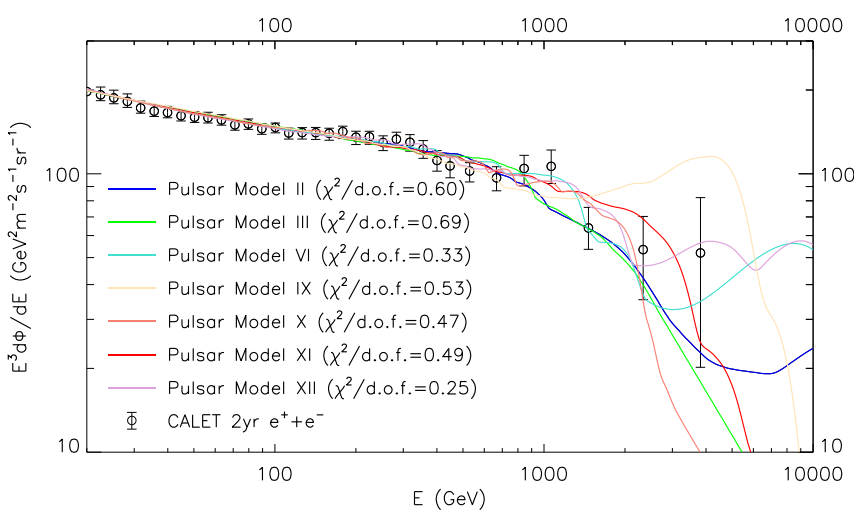

FIG. 11. Seven different predicted $e^{+}+e^{-}$fluxes from realizations that can fit the $C A L E T$ data and their $\chi^{2} /$ d.o.f.. Pulsar Model IX, which was excluded by DAMPE in Figure 10 can fit the $C A L E T$ data very well. Prominent features are also visible at the highest energies from powerful pulsars (models VI, IX, XI and XII).

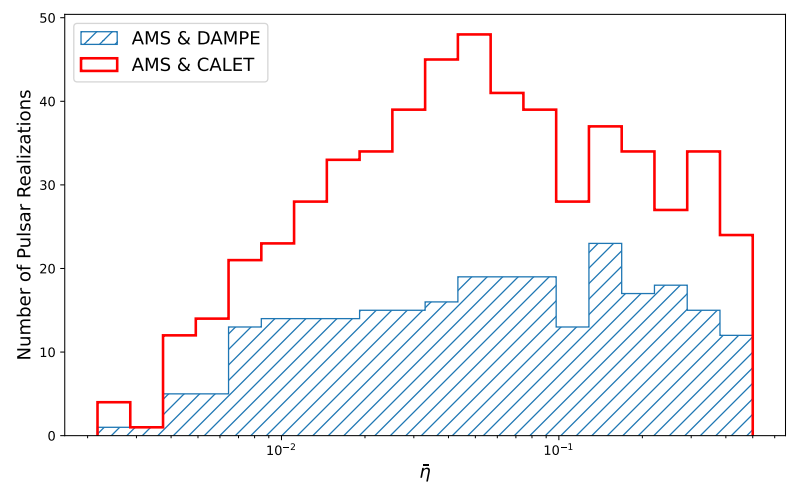

FIG. 12. The distribution of the fitted values for the averaged conversion efficiency $\bar{\eta}$ of pulsars' spin down power to cosmic rays. The y-axis shows the number of allowed Milky Way pulsars simulations within $2 \sigma$. Typical values for $\bar{\eta}$ are $\simeq 0.1$.

Finally, we use the fits to the DAMPE and CALET in combination with the $A M S$-02 fits, to test the overall conversion efficiency $\eta$ of pulsars' spin-down power to power in cosmic-ray electrons and positrons. In Figure 12, we show for the allowed pulsars simulations their fitted mean efficiency $\bar{\eta}$. We find that $\bar{\eta}$ is typically between 0.05 and 0.2 . Due to equipartition of energy in the pulsar's environments, we do not allow for $\bar{\eta}>0.5$ in our fits. This is also shown in Figure 12 . There is only a small number of pulsar simulations with $\bar{\eta}<0.01$. Recently, an independent analysis of [72] suggested a similar fraction of spin-down power to cosmic-ray electrons and positrons. We present the simulations that are within the $2 \sigma$ criterion, but note that even if we used the larger number of simulations that are within $3 \sigma$, the results would not differ.

\section{B. Results for $E \geq 5 \mathrm{GeV}$ fits}

In this section we include the $A M S-02$ positron fraction and positron flux measurements down to energies of $5 \mathrm{GeV}$ and discuss the relevant implications of the increased energy range. For the total $e^{+}+e^{-}$flux we still fit the data above $15 \mathrm{GeV}$. The presence of the feature at $\simeq 12 \mathrm{GeV}$ has an effect in the results of this section. Instead, in the discussion of Section IVA, we fitted energies $E>15 \mathrm{GeV}$, avoiding its impact on the Milky Way pulsar properties.

As we have discussed also in Section IVA, for each pulsars simulation, we consider a low energy extrapolation as well. We find that this low-energy extrapolation being included is more important than in Section IVA. where in fact for some ISM energy-loss assumptions the extrapolation starts at energies lower than $15 \mathrm{GeV}$. Even with the increased level of allowed low-energy flux uncertainty, many more simulations can be excluded by the data than in Section IVA. That is to be expected as the $A M S$-02 positron and electron fluxes at these energies have significantly smaller statistical errors. However, as we will show the presence of the feature around $12 \mathrm{GeV}$ also has an impact. Including the positron fraction and positron flux measurements from 5 to $15 \mathrm{GeV}$, only a handful of Milky Way pulsars simulations are within $2 \sigma$ agreement to the $A M S$-02 observations. For the remainder we focus on simulations that are within $3 \sigma$ and $5 \sigma$ from an expectation of $\chi^{2}$ of 1 per degree of freedom, i.e. simulations that are not excluded within $3 \sigma$ and $5 \sigma$. To demonstrate how more challenging it is to fit the $A M S$ 02 data at $E \geq 5 \mathrm{GeV}$ compared to $E>15 \mathrm{GeV}$ we include Figure 13. On the left part of that figure, we show the fraction of allowed simulations within $3 \sigma$ from the fits to $E>15 \mathrm{GeV}$ and on the right the equivalent fraction of allowed simulations within $3 \sigma$ from the fits to $E \geq 5 \mathrm{GeV}$. There is a dramatic decrease in the number of allowed simulations.

We test separately our simulations to each of the three $A M S$-02 measurements and present results that are consistent with all three (for more details see Section III B). We find that of the 7272 astrophysical realizations, 2831 can fit the AMS-02 positron-flux spectrum within $5 \sigma$. Of these 2831 realizations, only 37 (261) can also fit within $3 \sigma(5 \sigma)$ the $A M S-02$ positron fraction spectrum and the $e^{+}+e^{-}$spectrum. The positron fraction has by far the greatest impact in excluding simulations 6

We summarize in tables IV, $\mathrm{V}$ and $\mathrm{VI}$ the properties of the pulsars and the ISM needed to explain the $A M S$-02 observations. For every cell, we show the percentage of the pulsars simulations that are consistent within $3 \sigma$ and $5 \sigma$ (in parentheses) to the $A M S$-02 data. For instance, in Table IV of the simulations with the combination of

\footnotetext{
${ }^{6}$ Just fitting the the positron fraction, we find only 50 (325) pulsars simulations to it within $3 \sigma(5 \sigma)$
} 

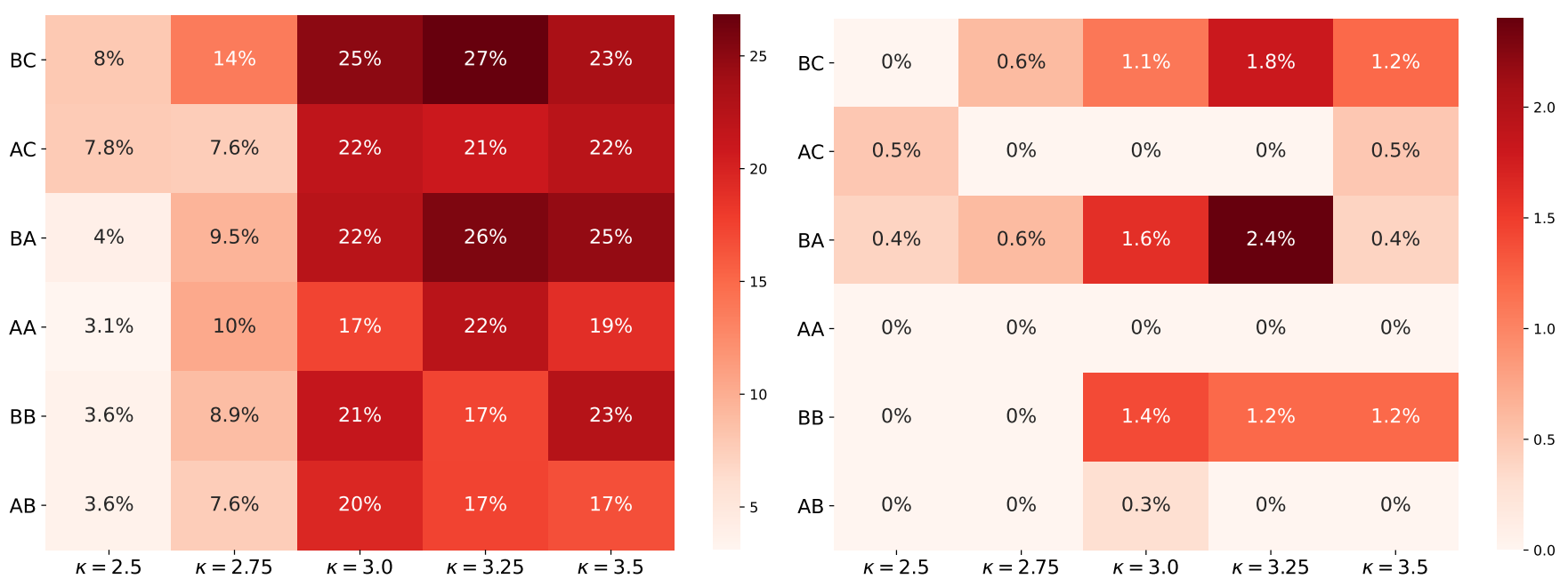

FIG. 13. Similarly to Figure 9 for given combination of pulsars' properties (each cell), we show the fraction of pulsar population simulations that are consistent within $3 \sigma$. On the left we have fitted the simulations to the $A M S$-02 observations from $E>15$ $\mathrm{GeV}$, and on the right to the $A M S-02$ observations from $E \geq 5 \mathrm{GeV}$ (see text for further details).

$\kappa=3.0$ and ISM assumptions "A2", only $2 \%(15 \%)$ are consistent within $3 \sigma(5 \sigma)$ to the $A M S-02$ data.

In Table IV], we show our results for the combination of the five choices of the braking index and the twelve choices of ISM propagation conditions. There is a clear preference for the ISM models "A2", "C2", "E2" and "F2" that predict the highest energy losses ( $b=8.02 \times 10^{-6} \mathrm{GeV}^{-1} \mathrm{kyrs}^{-1}$ in Table II). That strong preference is mostly the result of a spectral feature at $\simeq$ $12 \mathrm{GeV}$. The "A2", "C2", "E2" and "F2" without their low-energy extrapolation have a break close to that energy. We believe this feature likely suggests an additional source of positrons around 10-15 GeV, which may be a population of more distant pulsars closer to the inner spiral arm, around $5 \mathrm{kpc}$ away from us. A second reason why low ISM energy losses models perform worse once including the $5-15 \mathrm{GeV}$ data to our fits, is that these simulations predict fluxes that overshoot the observed spectra at high energies.

Our finding from Section IVA for a preference of a braking index $\kappa \geq 3.0$ remains to be the case even with the lower energy observations. In Table [IV] the choices $\kappa=2.5$ and $\kappa=2.75$ are almost entirely excluded (see also Table VI . Instead, for $\kappa=3.0$ there is a significant increase in the percentage of simulations in agreement to the data, followed by an even higher percentage for $\kappa=3.25$. For $\kappa=3.5$ we find similar results as for $\kappa=3.0$.

In Table V], we present as in Figure 8 our the results for the combination of injection index $n$ and $g(\eta)$ and the twelve ISM models. Our findings are similar to those presented in Section IVA with the difference that now there are practically no simulations allowed with $n \in[1.4,1.9]$ at $3 \sigma$ (first letter "A" along the raws). Again the choices of "CA", "CB" and "CC" are not presented as only one simulation was found to be within $5 \sigma$ to the data. Thus in our analysis, we can probe effectively the distribution properties of the injection index $n$. Yet, the stronger degeneracies on $g(\eta)$ are more difficult to further reduce with the lower energy data. At these low energies we observe the combined fluxes from thousands of pulsars and also the uncertainties associated with the cosmic ray secondaries become prominent. We note that the similarity on the derived properties of $n$ and $g(\eta)$ in the results of this section and Section IVA are to be expected. Varying the assumptions on $n$ and $g(\eta)$ affects the shape and magnitude of spectral features appearing at high energies and not between 5 and $15 \mathrm{GeV}$.

In Table VI. we present for completeness our results for the combination of the six choices for $n$ and $g(\eta)$, and the five choices for braking index. The domination of the choice "B", followed by choice "A", for $n$ that was described in the previous paragraphs is shown, and also the preference for braking index values $\kappa \geq 3$.

\section{DISCUSSION AND CONCLUSIONS}

In this paper we use recent cosmic-ray electron and positron observations from the $A M S-02, D A M P E$ and $C A L E T$ collaborations as a new handle to constrain the properties of Milky Way pulsars. Unlike electromagnetic spectrum observations that study individual objects, in our work we do not constrain the properties of any single pulsar. Instead, we constrain the properties of the general population of pulsars that can contribute to the observed cosmic-ray fluxes from $5 \mathrm{GeV}$ up to $5 \mathrm{TeV}$. As the observed electron/positron cosmic-ray energy increases the volume of possible sources decreases. This makes our analysis restricted to a smaller fraction of the local ISM volume at the highest energies.

We created simulations of the Milky Way pulsars that 


\begin{tabular}{c|cccccccccccc}
\hline & $\begin{array}{c}\mathrm{A} 1 \\
(\%)\end{array}$ & $\begin{array}{c}\mathrm{A} 2 \\
(\%)\end{array}$ & $\begin{array}{c}\mathrm{A} 3 \\
(\%)\end{array}$ & $\begin{array}{c}\mathrm{C} 1 \\
(\%)\end{array}$ & $\begin{array}{c}\mathrm{C} 2 \\
(\%)\end{array}$ & $\begin{array}{c}\mathrm{C} 3 \\
(\%)\end{array}$ & $\begin{array}{c}\mathrm{E} 1 \\
(\%)\end{array}$ & $\begin{array}{c}\mathrm{E} 2 \\
(\%)\end{array}$ & $\begin{array}{c}\mathrm{E} 3 \\
(\%)\end{array}$ & $\begin{array}{c}\mathrm{F} 1 \\
(\%)\end{array}$ & $\begin{array}{c}\mathrm{F} 2 \\
(\%)\end{array}$ & $\begin{array}{c}\mathrm{F} 3 \\
(\%)\end{array}$ \\
\hline \hline$\kappa=2.5$ & $0(0)$ & $0.8(4)$ & $0(0)$ & $0(0)$ & $0(0.8)$ & $0(0)$ & $0(0)$ & $0.8(2)$ & $0(0)$ & $0(0)$ & $0(2)$ & $0(0)$ \\
$\kappa=2.75$ & $0(0)$ & $0(7)$ & $0(0)$ & $0(0)$ & $1(3)$ & $0(0)$ & $0(1)$ & $0(2)$ & $0(0)$ & $0(0)$ & $1(6)$ & $0(0)$ \\
$\kappa=3.0$ & $0(1)$ & $2(15)$ & $0(0)$ & $0(0)$ & $2(11)$ & $0(0)$ & $0(0)$ & $3(11)$ & $0(0)$ & $0(0)$ & $0.4(4)$ & $0(0)$ \\
$\kappa=3.25$ & $0(1)$ & $1(16)$ & $0(0)$ & $0(3)$ & $6(18)$ & $0(0)$ & $0(1)$ & $1(20)$ & $0(0)$ & $0(0)$ & $2(10)$ & $0(0)$ \\
$\kappa=3.5$ & $0(0)$ & $1(11)$ & $0(0)$ & $0(0)$ & $3(15)$ & $0(0)$ & $0(0)$ & $0.6(11)$ & $0(0)$ & $0(0)$ & $0(8)$ & $0(0)$ \\
\hline \hline
\end{tabular}

TABLE IV. We show our results for the combination of the five choices of braking index $\kappa=2.5,2.75,3.0,3.25,3.5$ and the twelve choices of ISM propagation conditions modeled by "A1" to "F3" (see Table II). We give the fraction of pulsar population simulations that are consistent within $3 \sigma$ and $5 \sigma$ limits (in parentheses) to the $A M S$-02 positron fraction spectrum, the positron flux and the electron+positron flux (see text for details). For the combination of $\kappa=3$ and "C2" we produced 288 simulations to probe the remaining astrophysical parameters, of which $5(31)$ i.e. $\simeq 2 \%(11 \%)$ are allowed within $3 \sigma(5 \sigma)$.

\begin{tabular}{c|c|cccccccccccc}
\hline & & $\begin{array}{c}\mathrm{A} 1 \\
(\%)\end{array}$ & $\begin{array}{c}\mathrm{A} 2 \\
(\%)\end{array}$ & $\begin{array}{c}\mathrm{A} 3 \\
(\%)\end{array}$ & $\begin{array}{c}\mathrm{C} 1 \\
(\%)\end{array}$ & $\begin{array}{c}\mathrm{C} 2 \\
(\%)\end{array}$ & $\begin{array}{c}\mathrm{C} 3 \\
(\%)\end{array}$ & $\begin{array}{c}\mathrm{E} 1 \\
(\%)\end{array}$ & $\begin{array}{c}\mathrm{E} 2 \\
(\%)\end{array}$ & $\begin{array}{c}\mathrm{E} 3 \\
(\%)\end{array}$ & $\begin{array}{c}\mathrm{F} 1 \\
(\%)\end{array}$ & $\begin{array}{c}\mathrm{F} 2 \\
(\%)\end{array}$ & $\begin{array}{c}\mathrm{F} 3 \\
(\%)\end{array}$ \\
\hline \hline $\mathrm{BC}$ & $1.6 \leq n \leq 1.7, \zeta=1.29$ & $0(0)$ & $3(18)$ & $0(0)$ & $0(0)$ & $3(19)$ & $0(0)$ & $0(0)$ & $0.8(9)$ & $0(0)$ & $0(0)$ & $2(14)$ & $0(0)$ \\
$\mathrm{AC}$ & $1.4 \leq n \leq 1.9, \zeta=1.29$ & $0(2)$ & $0(13)$ & $0(0)$ & $0(0)$ & $1(8)$ & $0(0)$ & $0(0)$ & $1(4)$ & $0(0)$ & $0(0)$ & $0(4)$ & $0(0)$ \\
$\mathrm{BA}$ & $1.6 \leq n \leq 1.7, \zeta=1.47$ & $0(0)$ & $2(19)$ & $0(0)$ & $0(0)$ & $5(15)$ & $0(0)$ & $0(0)$ & $2(20)$ & $0(0)$ & $0(0)$ & $2(6)$ & $0(0)$ \\
$\mathrm{AA}$ & $1.4 \leq n \leq 1.9, \zeta=1.47$ & $0(1)$ & $0(10)$ & $0(0)$ & $0(0)$ & $0(8)$ & $0(0)$ & $0(0)$ & $0(10)$ & $0(0)$ & $0(0)$ & $0(2)$ & $0(0)$ \\
$\mathrm{BB}$ & $1.6 \leq n \leq 1.7, \zeta=2.85$ & $0(0)$ & $2(7)$ & $0(0)$ & $0(0)$ & $3(13)$ & $0(0)$ & $0(2)$ & $2(10)$ & $0(0)$ & $0(0)$ & $0(4)$ & $0(0)$ \\
$\mathrm{AB}$ & $1.4 \leq n \leq 1.9, \zeta=2.85$ & $0(0)$ & $0(2)$ & $0(0)$ & $0(2)$ & $0(2)$ & $0(0)$ & $0(0)$ & $1(7)$ & $0(0)$ & $0(0)$ & $0(1)$ & $0(0)$ \\
\hline \hline
\end{tabular}

TABLE V. As in Table IV] we present, for the combination of the six choices ("BC", "AC", "BA", "AA", "BB" and "AB") of $n$ and $g(\eta)$ and the twelve ISM models, the $\%$ fraction of pulsar simulations that are consistent within the $3 \sigma$ and $5 \sigma$ limits (in parentheses) to the $A M S$-02 cosmic-ray data.

lie within $4 \mathrm{kpc}$ from the location of the Sun. We have performed over $72 \times 10^{2}$ Milky Way pulsars simulations to account for i) the stochastic nature of the neutron stars' birth distribution in space and time, and uncertainties on the pulsars' birth rate, ii) uncertainties on the initial spin-down power distribution that Milky Way pulsars follow, iii) different assumptions on the evolution with time of the pulsars' spin-down power, iv) different assumptions on the cosmic-ray electron and positron fluxes that pulsars inject into the ISM and v) uncertainties on the propagation of these cosmic rays through the ISM and the Heliosphere before they get detected. The range of model parameter values that we explore is wide and varying among different assumptions can significantly affect the observed electron and positron fluxes as shown in Figures 2 and 3 . Each of our Milky Way pulsars simulations contains from $5 \times 10^{3}$ to $19 \times 10^{3}$ unique pulsars with ages up to $10 \mathrm{Myr}$, depending on the exact birth rate assumption. Within a given simulation, each pulsar has a unique location, age, initial spin-down power and spectral index of injected cosmic rays.

In performing our fits to the $A M S$-02, DAMPE and $C A L E T$ flux observations, we account also for the existence of cosmic-ray primary electrons from SNRs and secondary electrons and positrons produced in inelastic collisions taking place in the ISM. We also account for those fluxes' respective uncertainties. Examples of our fits are given in Figures 1, 4, 5, 10 and 11.

We find a strong preference for pulsars models with a spin-down braking index of $\kappa \geq 3.0$ (see Eq. 2 and Figures 7.9 and 13). Such a result is in contrast to observations of the about ten young pulsars, for which a reliable measurement of $\kappa<3.0$ has been made (with the exception of one [140]). As our analysis tests the averaged properties of much older pulsars than the electromagnetic measurements do, our results show that pulsars' breaking index evolves with time to larger values. This results in older pulsars losing their rotational energy in a slower manner than that predicted from the regular magnetic dipole radiation. Our results show a new way of studying the evolution of pulsars. With higher statistics in the future we expect that specific models on the pulsars' braking index evolution with time can be tested.

Furthermore, we find that pulsars inject into the ISM electrons and positrons with relatively similar cosmic-ray spectra that scale roughly as $d N / d E \propto E^{-1.6}$ up to $O(10)$ $\mathrm{TeV}$. Also, pulsars convert $O(10 \%)$ of their rotational energy into such cosmic rays (see Figure 12). Our conclusions are fairly robust to the exact birth rate of Milky Way pulsars and the exact local ISM assumptions. We still find a preference for larger pulsar birth rates and thicker diffusion zone ISM models.

Finally, when studying the lower energies we noticed that the $A M S$-02 positron measurements give a spectral feature at $\simeq 12 \mathrm{GeV}$. While some of our simulations can explain such a feature, its presence likely suggests a population of positron sources outside the volume of study, or of an entirely different origin. We leave the possible origin of such a feature to future studies.

We have made publicly available our Milky Way pulsars simulations in their pre-fitted format for the entire set. We have also provided the fitted fluxes from simulations that are in agreement with the $A M S$-02, $C A L E T$ and $D A M P E$ observations. These files can be found at 


\begin{tabular}{|c|c|c|c|c|c|c|}
\hline & & $\begin{array}{c}\kappa=2.5 \\
(\%)\end{array}$ & $\begin{array}{c}\kappa=2.75 \\
(\%)\end{array}$ & $\begin{array}{c}\kappa=3.0 \\
(\%)\end{array}$ & $\begin{array}{c}\kappa=3.25 \\
(\%)\end{array}$ & $\begin{array}{c}\kappa=3.5 \\
(\%)\end{array}$ \\
\hline$\overline{\mathrm{BC}}$ & $1.6 \leq n \leq 1.7, \zeta=1.29$ & $0(1)$ & 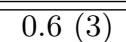 & $1(8)$ & $2(10)$ & $18(8)$ \\
\hline $\mathrm{AC}$ & $1.4 \leq n$ & $0.5(1)$ & $0(1$ & $0(4)$ & $0(2)$ & $0.5(3)$ \\
\hline $\mathrm{BA}$ & $1.6 \overline{\leq} n \leq 1.7, \zeta=$ & $0.4(3)$ & $0.6(4)$ & $2(7)$ & $2(12)$ & $0.4(7)$ \\
\hline AA & 1.4 & $0(0)$ & $0(0$ & $0(4)$ & $0(8)$ & $0(0.9)$ \\
\hline $\mathrm{BB}$ & $1.6 \overline{\leq} n \leq 1.7, \zeta=2.85$ & $0(0)$ & $0(2)$ & $1(4)$ & $1(4)$ & $1(8)$ \\
\hline $\mathrm{AB}$ & $1.4 \overline{\leq} n \overline{\leq} 1.9, \zeta=2.85$ & $0(0)$ & $0(0)$ & $0.3(2)$ & $0(2)$ & $0(2)$ \\
\hline
\end{tabular}

TABLE VI. Similar to the slices in parameter space given in Tables IV and $\mathrm{V}$, we show for the combination of the six choices of $n$ and $g(\eta)$ and the five choices for braking index $\kappa=2.5,2.75,3.0,3.25,3.5$, the \% fraction of pulsar population simulations that are consistent within $3 \sigma$ and $5 \sigma$ to the $A M S-02$ cosmic-ray data.

https://zenodo.org/record/5659004\#. YYqnbi-ZN0s. Acknowledgements: We thank Tanvi Karwal for useful discussions and Alexandros Kehagias for establish- ing this collaboration. IC acknowledges support from the NASA Michigan Space Grant Consortium, Grant No. 80NSSC20M0124.
[1] A. Hewish, S. J. Bell, J. D. H. Pilkington, P. F. Scott, and R. A. Collins, "Observation of a Rapidly Pulsating Radio Source," Nature (London) 217, 709-713 (1968)

[2] D. M. Gould and A. G. Lyne, "Multifrequency polarimetry of 300 radio pulsars," Mon. Not. R. Astron. Soc. 301, 235-260 (1998)

[3] I. H. Stairs, S. E. Thorsett, and F. Camilo, "Coherently Dedispersed Polarimetry of Millisecond Pulsars," Astrophys. J. Supp. 123, 627-638 (1999), arXiv:astro$\mathrm{ph} / 9810399$ [astro-ph].

[4] J. M. Weisberg et al., "Arecibo $1418 \mathrm{MHz}$ polarimetry of 98 pulsars: Full stokes profiles and morphological classifications," The Astrophysical Journal Supplement Series 121, 171-217 (1999)

[5] J. E. Everett and J. M. Weisberg, "Emission beam geometry of selected pulsars derived from average pulse polarization data," Astrophys. J. 553, 341 (2001) arXiv:astro-ph/0009266

[6] Maura A. McLaughlin and Joanna M. Rankin, "'Notches' in the average profiles of bright pulsars," Mon. Not. Roy. Astron. Soc. 351, 808 (2004), arXiv:astro-ph $/ 0311498$

[7] J. M. Weisberg et al., "Arecibo $430 \mathrm{MHz}$ pulsar polarimetry: Faraday rotation measures and morphological classifications," Astrophys. J. Suppl. 150, 317 (2004), arXiv:astro-ph/0310073

[8] R. N. Manchester, G. B. Hobbs, A. Teoh, and M. Hobbs, "The Australia Telescope National Facility Pulsar Catalogue," Astron. J. 129, 1993-2006 (2005), arXiv:astro-ph/0412641 [astro-ph]

[9] A. Sandberg and J. Sollerman, "Optical and infrared observations of the Crab Pulsar and its nearby knot," Astron. Astrophys. 504, 525-530 (2009), arXiv:0906.2065 [astro-ph.HE]

[10] R. P. Mignani et al., "The near-infrared detection of PSR B0540-69 and its nebula," Astron. Astrophys. 544, A100 (2012), arXiv:1204.6655 [astro-ph.HE]

[11] A. Danilenko et al., "Possible optical counterpart of PSR J1357-6429," Astron. Astrophys. 540, A28 (2012). arXiv:1202.0437 [astro-ph.SR]

[12] T. Schweizer et al., "Characterization of the optical and $\mathrm{X}$-ray properties of the north-western wisps in the Crab nebula," Mon. Not. R. Astron. Soc. 433, 3325-3335 (2013), arXiv:1301.1321 [astro-ph.HE]

[13] J. Sollerman, J. Selsing, P. M. Vreeswijk, P. Lundqvist, and A. Nyholm, "The optical and NIR spectrum of the Crab pulsar with X-shooter," Astron. Astrophys. 629, A140 (2019), arXiv:1906.09878 [astro-ph.HE].

[14] R. P. Mignani et al., "The First Ultraviolet Detection of the Large Magellanic Cloud Pulsar PSR B0540-69 and Its Multi-wavelength Properties," Astrophys. J. 871, 246 (2019), arXiv:1809.10805 [astro-ph.HE].

[15] F. Ambrosino et al., "Optical and ultraviolet pulsed emission from an accreting millisecond pulsar," Nature Astronomy 5, 552-559 (2021), arXiv:2102.11704 [astroph.HE]

[16 $\mid$ R. Giacconi, H. Gursky, E. Kellogg, E. Schreier, and H. Tananbaum, "Discovery of Periodic X-Ray Pulsations in Centaurus X-3 from UHURU," Astrophys. J. Lett. 167, L67 (1971)

[17] W. Becker and B. Aschenbach, "X-ray observations of neutron stars and pulsars: first results from xmmnewton," (2002), arXiv:astro-ph/0208466

[18] P. A. Gentile et al., "X-Ray Observations of Black Widow Pulsars," Astrophys. J. 783, 69 (2014) arXiv:1305.6799 [astro-ph.HE].

[19] S. Guillot et al., "NICER X-ray Observations of Seven Nearby Rotation-Powered Millisecond Pulsars," Astrophys. J. Lett. 887, L27 (2019), arXiv:1912.05708 [astroph.HE|

[20] Jiaqi Zhao, Yue Zhao, and Craig O. Heinke, "Chandra and HST Studies of Six Millisecond Pulsars in the Globular Cluster M13," Mon. Not. Roy. Astron. Soc. 502, 1596-1604 (2021), arXiv:2101.07986 [astro-ph.HE].

[21] M. P. Ulmer, "Gamma-Ray Observations of Pulsars," Astrophys. J. Supp. 90, 789 (1994).

[22] A. A. Abdo et al., "The First Fermi Large Area Telescope Catalog of Gamma-ray Pulsars," Astrophys. J. Supp. 187, 460-494 (2010), arXiv:0910.1608 [astro-ph.HE]

[23] A. A. Abdo et al. (Fermi-LAT), "The Second Fermi Large Area Telescope Catalog of Gamma-ray Pulsars," Astrophys. J. Suppl. 208, 17 (2013), arXiv:1305.4385 astro-ph.HE] 
[24] Rolf Bühler and Roger Blandford, "The surprising Crab pulsar and its nebula: A review," Rept. Prog. Phys. 77, 066901 (2014), arXiv:1309.7046 [astro-ph.HE]

[25] Ilias Cholis, Dan Hooper, and Tim Linden, "A New Determination of the Spectra and Luminosity Function of Gamma-Ray Millisecond Pulsars," (2014), arXiv:1407.5583 [astro-ph.HE]

[26] S. Abdollahi et al. (Fermi-LAT), "Fermi Large Area Telescope Fourth Source Catalog," Astrophys. J. Suppl. 247, 33 (2020), arXiv:1902.10045 [astro-ph.HE]

[27] A. A. Abdo et al., "Milagro Observations of Multi$\mathrm{TeV}$ Emission from Galactic Sources in the Fermi Bright Source List," Astrophys. J. Lett. 700, L127-L131 (2009), arXiv:0904.1018 [astro-ph.HE]

[28] S. Ansoldi et al. (MAGIC), "Teraelectronvolt pulsed emission from the Crab pulsar detected by MAGIC," Astron. Astrophys. 585, A133 (2016), arXiv:1510.07048 astro-ph.HE|

[29] H. Abdalla et al. (HESS), "The population of TeV pulsar wind nebulae in the H.E.S.S. Galactic Plane Survey," Astron. Astrophys. 612, A2 (2018), arXiv:1702.08280 [astro-ph.HE]

[30] A. U. Abeysekara et al., "The 2HWC HAWC Observatory Gamma Ray Catalog," Astrophys. J. 843, 40 (2017), arXiv:1702.02992 [astro-ph.HE]

[31] A. U. Abeysekara et al. (HAWC), "Multiple Galactic Sources with Emission Above $56 \mathrm{TeV}$ Detected by HAWC," Phys. Rev. Lett. 124, 021102 (2020), arXiv:1909.08609 [astro-ph.HE]

[32] J. A. Gil and J. K. Snakowski, "Curvature radiation and the core emission of pulsars." Astron. Astrophys. 234, 237-242 (1990).

[33] P. F. Wang, C. Wang, and J. L. Han, "Curvature radiation in rotating pulsar magnetosphere," in Neutron Stars and Pulsars: Challenges and Opportunities after 80 years Vol. 291, edited by Joeri van Leeuwen (2013) pp. 552-554.

[34] Luca Del Zanna, Delia Volpi, Elena Amato, and Niccolo' Bucciantini, "Simulated synchrotron emission from pulsar wind nebulae," Astron. Astrophys. 453, 621 (2006), arXiv:astro-ph/0603080

[35] Oliver Porth, Serguei S. Komissarov, and Rony Keppens, "Three-Dimensional Magnetohydrodynamic Simulations of the Crab Nebula," Mon. Not. Roy. Astron. Soc. 438, 278-306 (2014), arXiv:1310.2531 [astroph.HE|.

[36] A. M. Atoyan and F. A. Aharonian, "On the mechanisms of gamma radiation in the Crab Nebula," Mon. Not. R. Astron. Soc. 278, 525-541 (1996)

[37] M. Meyer, D. Horns, and H. S. Zechlin, "The Crab Nebula as a standard candle in very high-energy astrophysics," Astron. Astrophys. 523, A2 (2010), arXiv:1008.4524 [astro-ph.HE]

[38] Oleg Kargaltsev, Benoit Cerutti, Yuri Lyubarsky, and Edoardo Striani, "Pulsar-Wind Nebulae: Recent Progress in Observations and Theory," Space Sci. Rev. 191, 391-439 (2015) arXiv:1507.03662 [astro-ph.HE]

[39] Jonathan Arons, "Pulsars as Gamma-Rays Sources: Nebular Shocks and Magnetospheric Gaps," Space Science Rev. 75, 235-255 (1996).

[40] Dmitry Malyshev, Ilias Cholis, and Joseph Gelfand, "Pulsars versus Dark Matter Interpretation of ATIC/PAMELA," Phys. Rev. D80, 063005 (2009). arXiv:0903.1310 [astro-ph.HE]
[41] Igor V. Moskalenko, Andrew W. Strong, Jonathan F. Ormes, and Marius S. Potgieter, "Secondary antiprotons and propagation of cosmic rays in the galaxy and heliosphere," Astrophys. J. 565, 280-296 (2002) arXiv:astro-ph/0106567 [astro-ph]

[42] Michael Kachelriess, Igor V. Moskalenko, and Sergey S. Ostapchenko, "New calculation of antiproton production by cosmic ray protons and nuclei," Astrophys. J. 803, 54 (2015), arXiv:1502.04158 [astro-ph.HE]

[43] http://galprop.stanford.edu/.,.

[44] A. W. Strong, "Recent extensions to GALPROP," (2015), arXiv:1507.05020 [astro-ph.HE]

[45] Carmelo Evoli, Daniele Gaggero, Dario Grasso, and Luca Maccione, "Cosmic-Ray Nuclei, Antiprotons and Gamma-rays in the Galaxy: a New Diffusion Model," JCAP 0810, 018 (2008), arXiv:0807.4730 [astroph] $\% \%$ CITATION $=$ ARXIV $: 0807.4730 ; \% \%$.

[46] http://dragon.hepforge.org,.

[47] Carmelo Evoli, Ilias Cholis, Dario Grasso, Luca Maccione, and Piero Ullio, "Antiprotons from dark matter annihilation in the Galaxy: astrophysical uncertainties," Phys. Rev. D85, 123511 (2012), arXiv:1108.0664 [astroph.HE]

[48] Miguel Pato, Dan Hooper, and Melanie Simet, "Pinpointing Cosmic Ray Propagation With The AMS-02 Experiment," JCAP 1006, 022 (2010), arXiv:1002.3341 [astro-ph.HE]

[49] Ilias Cholis, YiMing Zhong, Sam McDermott, and Joseph Surdutovich, "In preparation," (2021).

[50] M. Aguilar et al. (AMS), "Precision Measurement of the Helium Flux in Primary Cosmic Rays of Rigidities 1.9 GV to $3 \mathrm{TV}$ with the Alpha Magnetic Spectrometer on the International Space Station," Phys. Rev. Lett. 115, 211101 (2015)

[51] M. Aguilar et al. (AMS), "Observation of the Identical Rigidity Dependence of He, C, and O Cosmic Rays at High Rigidities by the Alpha Magnetic Spectrometer on the International Space Station," Phys. Rev. Lett. 119, 251101 (2017).

[52] M. Aguilar et al. (AMS), "Observation of New Properties of Secondary Cosmic Rays Lithium, Beryllium, and Boron by the Alpha Magnetic Spectrometer on the International Space Station," Phys. Rev. Lett. 120, 021101 (2018)

[53] O. Adriani et al. (PAMELA), "Cosmic-Ray Positron Energy Spectrum Measured by PAMELA," Phys. Rev. Lett. 111, 081102 (2013), arXiv:1308.0133 [astroph.HE]

[54] L. Accardo et al. (AMS), "High Statistics Measurement of the Positron Fraction in Primary Cosmic Rays of 0.5-500 GeV with the Alpha Magnetic Spectrometer on the International Space Station," Phys. Rev. Lett. 113, 121101 (2014)

[55] M. Aguilar et al. (AMS), "Towards Understanding the Origin of Cosmic-Ray Electrons," Phys. Rev. Lett. 122, 101101 (2019)

[56] M. Aguilar et al. (AMS), "The Alpha Magnetic Spectrometer (AMS) on the international space station: Part II - Results from the first seven years," Phys. Rept. 894, 1-116 (2021)

[57] A. K. Harding and R. Ramaty, "The Pulsar Contribution to Galactic Cosmic Ray Positrons," International Cosmic Ray Conference 2, 92 (1987).

[58] A. M. Atoyan, F. A. Aharonian, and H. J. Völk, "Elec- 
trons and positrons in the galactic cosmic rays," Phys. Rev. D 52, 3265-3275 (1995).

[59] F. A. Aharonian, A. M. Atoyan, and H. J. Voelk, "High energy electrons and positrons in cosmic rays as an indicator of the existence of a nearby cosmic tevatron," Astron. Astrophys. 294, L41-L44 (1995).

[60] Dan Hooper, Pasquale Blasi, and Pasquale Dario Serpico, "Pulsars as the Sources of High Energy Cosmic Ray Positrons," JCAP 0901, 025 (2009). arXiv:0810.1527 [astro-ph],

[61] Hasan Yuksel, Matthew D. Kistler, and Todor Stanev, "TeV Gamma Rays from Geminga and the Origin of the GeV Positron Excess," Phys. Rev. Lett. 103, 051101 (2009), arXiv:0810.2784 [astro-ph].

[62] Stefano Profumo, "Dissecting cosmic-ray electronpositron data with Occam's Razor: the role of known Pulsars," Central Eur. J. Phys. 10, 1-31 (2011) arXiv:0812.4457 [astro-ph],

[63] Norita Kawanaka, Kunihito Ioka, and Mihoko M. Nojiri, "Cosmic-Ray Electron Excess from Pulsars is Spiky or Smooth?: Continuous and Multiple Electron/Positron injections," Astrophys. J. 710, 958-963 (2010), arXiv:0903.3782 [astro-ph.HE]

[64] D. Grasso et al. (Fermi-LAT), "On possible interpretations of the high energy electron-positron spectrum measured by the Fermi Large Area Telescope," Astropart. Phys. 32, 140-151 (2009), arXiv:0905.0636 [astro-ph.HE]

[65] Tim Linden and Stefano Profumo, "Probing the Pulsar Origin of the Anomalous Positron Fraction with AMS02 and Atmospheric Cherenkov Telescopes," Astrophys.J. 772, 18 (2013), arXiv:1304.1791 [astro-ph.HE]

[66] Ilias Cholis and Dan Hooper, "Dark Matter and Pulsar Origins of the Rising Cosmic Ray Positron Fraction in Light of New Data From AMS," Phys. Rev. D88, 023013 (2013), arXiv:1304.1840 [astro-ph.HE]

[67] Q. Yuan et al., "Implications of the AMS-02 positron fraction in cosmic rays," Astropart. Phys. 60, 1-12 (2015), arXiv:1304.1482 [astro-ph.HE]

[68] Peng-Fei Yin, Zhao-Huan Yu, Qiang Yuan, and XiaoJun Bi, "Pulsar interpretation for the AMS-02 result," Phys. Rev. D88, 023001 (2013), arXiv:1304.4128 [astroph.HE]

[69] Ilias Cholis, Tanvi Karwal, and Marc Kamionkowski, "Studying the Milky Way pulsar population with cosmic-ray leptons," Phys. Rev. D 98, 063008 (2018), arXiv:1807.05230 [astro-ph.HE]

[70] Carmelo Evoli, Elena Amato, Pasquale Blasi, and Roberto Aloisio, "Galactic factories of cosmic-ray electrons and positrons," Phys. Rev. D 103, 083010 (2021), arXiv:2010.11955 [astro-ph.HE]

[71] Silvia Manconi, Mattia Di Mauro, and Fiorenza Donato, "Detection of a $\gamma$-ray halo around Geminga with the Fermi-LAT and implications for the positron flux," PoS ICRC2019, 580 (2021).

[72] Luca Orusa, Silvia Manconi, Fiorenza Donato, and Mattia Di Mauro, "Constraining positron emission from pulsar populations with AMS-02 data," (2021), arXiv:2107.06300 [astro-ph.HE]

[73] Pasquale Blasi, "The origin of the positron excess in cosmic rays," Phys. Rev. Lett. 103, 051104 (2009), arXiv:0903.2794 [astro-ph.HE]

[74] Philipp Mertsch and Subir Sarkar, "Testing astrophysical models for the PAMELA positron excess with cos- mic ray nuclei," Phys. Rev. Lett. 103, 081104 (2009) arXiv:0905.3152 [astro-ph.HE].

[75] Markus Ahlers, Philipp Mertsch, and Subir Sarkar, "On cosmic ray acceleration in supernova remnants and the FERMI/PAMELA data," Phys. Rev. D80, 123017 (2009), arXiv:0909.4060 [astro-ph.HE]

[76] Pasquale Blasi and Pasquale D. Serpico, "Highenergy antiprotons from old supernova remnants," Phys.Rev.Lett. 103, 081103 (2009), arXiv:0904.0871 [astro-ph.HE].

[77] Norita Kawanaka, Kunihito Ioka, Yutaka Ohira, and Kazumi Kashiyama, "TeV Electron Spectrum for Probing Cosmic-Ray Escape from a Supernova Remnant," Astrophys. J. 729, 93 (2011), arXiv:1009.1142 [astroph.HE]

[78] Yutaka Fujita, Kazunori Kohri, Ryo Yamazaki, and Kunihito Ioka, "Is the PAMELA anomaly caused by the supernova explosions near the Earth?" Phys. Rev. D80, 063003 (2009), arXiv:0903.5298 [astro-ph.HE].

[79] Ilias Cholis and Dan Hooper, "Constraining the origin of the rising cosmic ray positron fraction with the boron-to-carbon ratio," Phys. Rev. D89, 043013 (2014), arXiv:1312.2952 [astro-ph.HE]

[80] Philipp Mertsch and Subir Sarkar, "AMS-02 data confront acceleration of cosmic ray secondaries in nearby sources," Phys. Rev. D90, 061301 (2014), arXiv:1402.0855 [astro-ph.HE].

[81] M. Di Mauro, F. Donato, N. Fornengo, R. Lineros, and A. Vittino, "Interpretation of AMS-02 electrons and positrons data," JCAP 1404, 006 (2014), arXiv:1402.0321 [astro-ph.HE]

[82] Kazunori Kohri, Kunihito Ioka, Yutaka Fujita, and Ryo Yamazaki, "Can we explain AMS-02 antiproton and positron excesses simultaneously by nearby supernovae without pulsars or dark matter?" PTEP 2016, 021E01 (2016), arXiv:1505.01236 [astro-ph.HE]

[83] Philipp Mertsch, "Stochastic cosmic ray sources and the TeV break in the all-electron spectrum," JCAP 11, 045 (2018), arXiv:1809.05104 [astro-ph.HE].

[84] Ilias Cholis, Dan Hooper, and Tim Linden, "Possible Evidence for the Stochastic Acceleration of Secondary Antiprotons by Supernova Remnants," Phys. Rev. D95, 123007 (2017), arXiv:1701.04406 [astro-ph.HE]

[85] Nicola Tomassetti and Alberto Oliva, "Production and acceleration of antinuclei in supernova shockwaves," Astrophys. J. 844, L26 (2017), arXiv:1707.06915 [astroph.HE]

[86] Lars Bergstrom, Torsten Bringmann, and Joakim Edsjo, "New Positron Spectral Features from Supersymmetric Dark Matter - a Way to Explain the PAMELA Data?" Phys. Rev. D78, 103520 (2008), arXiv:0808.3725 [astro-ph].

[87] Marco Cirelli and Alessandro Strumia, "Minimal Dark Matter predictions and the PAMELA positron excess," Proceedings, 7th International Workshop on the Identification of Dark Matter (IDM 2008): Stockholm, Sweden, August 18-22, 2008, PoS IDM2008, 089 (2008), arXiv:0808.3867 [astro-ph].

[88] Ilias Cholis, Lisa Goodenough, Dan Hooper, Melanie Simet, and Neal Weiner, "High Energy Positrons From Annihilating Dark Matter," Phys. Rev. D80, 123511 (2009), arXiv:0809.1683 [hep-ph]

[89] Marco Cirelli, Mario Kadastik, Martti Raidal, and Alessandro Strumia, "Model-independent implications 
of the e+-, anti-proton cosmic ray spectra on properties of Dark Matter," Nucl. Phys. B813, 1-21 (2009), [Addendum: Nucl. Phys.B873,530(2013)], arXiv:0809.2409 [hep-ph].

[90] Ann E. Nelson and Christopher Spitzer, "Slightly NonMinimal Dark Matter in PAMELA and ATIC," JHEP 10, 066 (2010), arXiv:0810.5167 [hep-ph].

191] Nima Arkani-Hamed, Douglas P. Finkbeiner, Tracy R. Slatyer, and Neal Weiner, "A Theory of Dark Matter," Phys. Rev. D79, 015014 (2009), arXiv:0810.0713 [hepph].

[92] Ilias Cholis, Douglas P. Finkbeiner, Lisa Goodenough, and Neal Weiner, "The PAMELA Positron Excess from Annihilations into a Light Boson," JCAP 0912, 007 (2009), arXiv:0810.5344 [astro-ph].

[93] Ilias Cholis, Gregory Dobler, Douglas P. Finkbeiner, Lisa Goodenough, and Neal Weiner, "The Case for a 700+ GeV WIMP: Cosmic Ray Spectra from ATIC and PAMELA," Phys. Rev. D80, 123518 (2009), arXiv:0811.3641 [astro-ph].

[94] Roni Harnik and Graham D. Kribs, "An Effective Theory of Dirac Dark Matter," Phys. Rev. D79, 095007 (2009), arXiv:0810.5557 [hep-ph].

[95] Patrick J. Fox and Erich Poppitz, "Leptophilic Dark Matter," Phys. Rev. D79, 083528 (2009) arXiv:0811.0399 [hep-ph]

[96] Maxim Pospelov and Adam Ritz, "Astrophysical Signatures of Secluded Dark Matter," Phys. Lett. B671, 391-397 (2009), arXiv:0810.1502 [hep-ph]

[97] John David March-Russell and Stephen Mathew West, "WIMPonium and Boost Factors for Indirect Dark Matter Detection," Phys. Lett. B676, 133-139 (2009), arXiv:0812.0559 [astro-ph].

[98] Spencer Chang and Lisa Goodenough, "Charge Asymmetric Cosmic Ray Signals From Dark Matter Decay," Phys. Rev. D84, 023524 (2011), arXiv:1105.3976 [hep$\mathrm{ph}]$.

[99] Keith R. Dienes, Jason Kumar, and Brooks Thomas, "Dynamical Dark Matter and the positron excess in light of AMS results," Phys. Rev. D88, 103509 (2013), arXiv:1306.2959 [hep-ph]

[100] Douglas P. Finkbeiner and Neal Weiner, "Exciting Dark Matter and the INTEGRAL/SPI $511 \mathrm{keV}$ signal," Phys. Rev. D76, 083519 (2007), arXiv:astro$\mathrm{ph} / 0702587$ [astro-ph].

[101] Joachim Kopp, "Constraints on dark matter annihilation from AMS-02 results," Phys. Rev. D88, 076013 (2013), arXiv:1304.1184 [hep-ph].

[102] P. S. Bhupal Dev, Dilip Kumar Ghosh, Nobuchika Okada, and Ipsita Saha, "Neutrino Mass and Dark Matter in light of recent AMS-02 results," Phys. Rev. D89, 095001 (2014), arXiv:1307.6204 [hep-ph].

[103] Michael Klasen, Martin Pohl, and Günter Sigl, "Indirect and direct search for dark matter," Prog. Part. Nucl. Phys. 85, 1-32 (2015), arXiv:1507.03800 [hep-ph].

[104] Qiang Yuan and Lei Feng, "Dark Matter Particle Explorer observations of high-energy cosmic ray electrons plus positrons and their physical implications," Sci. China Phys. Mech. Astron. 61, 101002 (2018), arXiv:1807.11638 [astro-ph.HE].

[105] Xudong Sun and Ben-Zhong Dai, "Dark matter annihilation into leptons through gravity portals," JHEP 04, 108 (2021), arXiv:2008.02994 [hep-ph]

[106] Tracy R. Slatyer, Nikhil Padmanabhan, and Douglas P.
Finkbeiner, "CMB Constraints on WIMP Annihilation: Energy Absorption During the Recombination Epoch," Phys. Rev. D80, 043526 (2009), arXiv:0906.1197 [astroph.CO]

[107] C. Evoli, M. Valdes, A. Ferrara, and N. Yoshida, "Energy deposition by weakly interacting massive particles: a comprehensiv e study," Mon. Not. Roy. Astron. Soc. 422, 420-433 (2012)

[108 Mathew S. Madhavacheril, Neelima Sehgal, and Tracy R. Slatyer, "Current Dark Matter Annihilation Constraints from CMB and Low-Redshift Data," Phys. Rev. D89, 103508 (2014), arXiv:1310.3815 [astroph.CO].

[109] P. A. R. Ade et al. (Planck), "Planck 2015 results. XIII. Cosmological parameters," Astron. Astrophys. 594, A13 (2016), arXiv:1502.01589 [astro-ph.CO]

[110] Tracy R. Slatyer, "Indirect dark matter signatures in the cosmic dark ages. I. Generalizing the bound on s-wave dark matter annihilation from Planck results," Phys. Rev. D93, 023527 (2016), arXiv:1506.03811 [hep-ph]

111| Vivian Poulin, Pasquale D. Serpico, and Julien Lesgourgues, "A fresh look at linear cosmological constraints on a decaying dark matter component," JCAP 1608, 036 (2016), arXiv:1606.02073 [astro-ph.CO]

[112] Maryam Tavakoli, Ilias Cholis, Carmelo Evoli, and Piero Ullio, "Constraints on dark matter annihilations from diffuse gamma-ray emission in the Galaxy," JCAP 1401, 017 (2014), arXiv:1308.4135 [astro-ph.HE]

[113] Alex Geringer-Sameth, Savvas M. Koushiappas, and Matthew G. Walker, "Comprehensive search for dark matter annihilation in dwarf galaxies," Phys. Rev. D91, 083535 (2015), arXiv:1410.2242 [astro-ph.CO]

[114] M. Ackermann et al. (Fermi-LAT), "Searching for Dark Matter Annihilation from Milky Way Dwarf Spheroidal Galaxies with Six Years of Fermi Large Area Telescope Data," Phys. Rev. Lett. 115, 231301 (2015), arXiv:1503.02641 [astro-ph.HE]

[115] GEORGE R. BLUMENTHAL and ROBERT J. GOULD, "Bremsstrahlung, synchrotron radiation, and compton scattering of high-energy electrons traversing dilute gases," Rev. Mod. Phys. 42, 237-270 (1970).

[116] Payel Mukhopadhyay and Tim Linden, "Self-Generated Cosmic-Ray Turbulence Can Explain the Morphology of TeV Halos," (2021), arXiv:2111.01143 [astro-ph.HE].

[117] P. M. Dragicevich, D. G. Blair, and R. R. Burman, "Why are supernovae in our Galaxy so frequent?" Mon. Not. R. Astron. Soc. 302, 693-699 (1999).

[118] Natasa Vranesevic et al., "Pulsar birthrate from Parkes multi-beam survey," IAU Symposium 218: Young Neutron Stars and Their Environment Sydney, Australia, July 14-17, 2003, Astrophys. J. 617, L139-L142 (2004), arXiv:astro-ph/0310201 [astro-ph]

[119] Claude-Andre Faucher-Giguere and Victoria M. Kaspi, "Birth and evolution of isolated radio pulsars," Astrophys. J. 643, 332-355 (2006), arXiv:astro-ph/0512585 [astro-ph]

[120] D. R. Lorimer et al., "The Parkes multibeam pulsar survey: VI. Discovery and timing of 142 pulsars and a Galactic population analysis," Mon. Not. Roy. Astron. Soc. 372, 777-800 (2006), arXiv:astro-ph/0607640 [astro-ph].

[121] E. F. Keane and M. Kramer, "On the birthrates of Galactic neutron stars," Mon. Not. Roy. Astron. Soc. 391, 2009 (2008), arXiv:0810.1512 [astro-ph] 
[122] Ilias Cholis, Tanvi Karwal, and Marc Kamionkowski, "Features in the Spectrum of Cosmic-Ray Positrons from Pulsars," Phys. Rev. D 97, 123011 (2018), arXiv:1712.00011 [astro-ph.HE]

[123] Ottavio Fornieri, Daniele Gaggero, and Dario Grasso, "Features in cosmic-ray lepton data unveil the properties of nearby cosmic accelerators," JCAP 02, 009 (2020), arXiv:1907.03696 [astro-ph.HE]

[124] R. N. Manchester et al., "The Parkes Multibeam Pulsar Survey. 1. Observing and data analysis systems, discovery and timing of 100 pulsars," Mon. Not. Roy. Astron. Soc. 328, 17 (2001), arXiv:astro-ph/0106522 [astro-ph]

[125] Dunc R. Lorimer, "The galactic population and birth rate of radio pulsars," IAU Symposium 218: Young Neutron Stars and Their Environment Sydney, Australia, July 14-17, 2003, (2003), [IAU Symp.218,105(2004)], arXiv:astro-ph/0308501 [astro-ph].

[126] F. Pacini and M. Salvati, "On the Evolution of Supernova Remnants. Evolution of the Magnetic Field, Particles, Content, and Luminosity," Astrophys. J. 186, 249266 (1973)

[127] R N Manchester, G B Hobbs, A Teoh, and M Hobbs, "The Australia Telescope National Facility pulsar catalogue," Astron. J. 129, 1993 (2005), arXiv:astro$\mathrm{ph} / 0412641$ [astro-ph]

[128 http://www.atnf.csiro.au/research/pulsar/psrcat,.

[129] Ioannis Contopoulos and Anatoly Spitkovsky, "Revised pulsar spindown," Astrophys. J. 643, 1139-1145 (2006). arXiv:astro-ph/0512002.

[130] Oliver Q. Hamil, Nick J. Stone, and Jirina R. Stone, "Braking Index of Isolated Pulsars II: A novel two-dipole model of pulsar magnetism," Phys. Rev. D 94, 063012 (2016), arXiv:1608.01375 [astro-ph.HE]

[131] Roger D. Blandford and Roger W. Romani, "On the interpretation of pulsar braking indices." Mon. Not. R. Astron. Soc. 234, 57P-60 (1988)

[132] Peter Goldreich and Andreas Reisenegger, "Magnetic Field Decay in Isolated Neutron Stars," Astrophys. J. 395, 250 (1992)

[133] Thomas M. Tauris and Sushan Konar, "Torque decay in the pulsar ( $\mathrm{p}, \mathrm{p}$ _dot) diagram. effects of crustal ohmic dissipation and allignment," Astron. Astrophys. 376, 543 (2001), arXiv:astro-ph/0101531

[134] Pablo Marchant, Andreas Reisenegger, Juan Alejandro Valdivia, and Jaime H. Hoyos, "Stability of Hall equilibria in neutron star crusts," Astrophys. J. 796, 94 (2014), arXiv:1410.5833 [astro-ph.HE]

[135] Zhi-Fu Gao, Na Wang, Hao Shan, Xiang-Dong Li, and Wei Wang, "The Dipole Magnetic Field and Spindown Evolutions of the High Braking Index Pulsar PSR J1640-4631," Astrophys. J. 849, 19 (2017), arXiv:1709.03459 [astro-ph.HE]

[136] Andrei P. Igoshev, Sergei B. Popov, and Rainer Hollerbach, "Evolution of neutron star magnetic fields," (2021), arXiv:2109.05584 [astro-ph.HE]

[137] H. Heiselberg and M. Hjorth-Jensen, "Phase transitions in rotating neutron stars," Phys. Rev. Lett. 80, 54855488 (1998), arXiv:astro-ph/9801187.

[138] P. S. Koliogiannis and Ch. C. Moustakidis, "Effects of the equation of state on the bulk properties of maximally-rotating neutron stars," Phys. Rev. C 101, 015805 (2020), arXiv:1907.13375 [nucl-th].

[139] O. Hamil, J. R. Stone, M. Urbanec, and G. Urbancová, "Braking index of isolated pulsars," Phys. Rev. D 91,
063007 (2015), arXiv:1608.01383 [astro-ph.HE]

[140] R. F. Archibald et al., "A High Braking Index for a Pulsar," Astrophys. J. Lett. 819, L16 (2016), arXiv:1603.00305 [astro-ph.HE].

[141] A. Parthasarathy et al., "Timing of young radio pulsars - II. Braking indices and their interpretation," Mon. Not. Roy. Astron. Soc. 494, 2012-2026 (2020) arXiv:2003.13303 [astro-ph.HE].

[142] A. A. Abdo et al., "Milagro Observations of TeV Emission from Galactic Sources in the Fermi Bright Source List," Astrophys. J. 700, L127-L131 (2009), [Erratum: Astrophys. J.703,L185(2009)], arXiv:0904.1018 [astroph.HE]

[143] A. U. Abeysekara et al. (HAWC), "Extended gammaray sources around pulsars constrain the origin of the positron flux at Earth," Science 358, 911-914 (2017), arXiv:1711.06223 [astro-ph.HE].

[144] A. Kolmogorov, "The Local Structure of Turbulence in Incompressible Viscous Fluid for Very Large Reynolds' Numbers," Akademiia Nauk SSSR Doklady 30, 301-305 (1941).

[145] Robert H. Kraichnan, "Inertial Ranges in TwoDimensional Turbulence," Physics of Fluids 10, 14171423 (1967)

[146] R. Trotta, G. Johannesson, I. V. Moskalenko, T. A. Porter, R. Ruiz de Austri, and A. W. Strong, "Constraints on cosmic-ray propagation models from a global Bayesian analysis," Astrophys. J. 729, 106 (2011), arXiv:1011.0037 [astro-ph.HE]

[147] O. Klein and T. Nishina, "Über die Streuung von Strahlung durch freie Elektronen nach der neuen relativistischen Quantendynamik von Dirac," Zeitschrift fur Physik 52, 853-868 (1929).

[148] George R. Blumenthal and Robert J. Gould, "Bremsstrahlung, Synchrotron Radiation, and Compton Scattering of High-Energy Electrons Traversing Dilute Gases," Reviews of Modern Physics 42, 237-271 (1970)

[149] E. S. Seo and V. S. Ptuskin, "Stochastic Reacceleration of Cosmic Rays in the Interstellar Medium," Astrophys. J. 431, 705 (1994)

[150] A. W. Strong and I. V. Moskalenko, "Propagation of cosmic-ray nucleons in the Galaxy," Astrophys. J. 509, 212-228 (1998), arXiv:astro-ph/9807150\%\% CITATION $=$ ASTRO-PH $/ 9807150 ; \% \%$.

[151] Troy A. Porter, Gudlaugur Johannesson, and Igor V. Moskalenko, "High-Energy Gamma Rays from the Milky Way: Three-Dimensional Spatial Models for the Cosmic-Ray and Radiation Field Densities in the Interstellar Medium," Astrophys. J. 846, 67 (2017), arXiv:1708.00816 [astro-ph.HE]

[152] L. J. Gleeson and W. I. Axford, "Solar Modulation of Galactic Cosmic Rays," Astrophys. J. 154, 1011 (1968).

[153] Ilias Cholis, Dan Hooper, and Tim Linden, "A Predictive Analytic Model for the Solar Modulation of Cosmic Rays," Phys. Rev. D93, 043016 (2016), arXiv:1511.01507 [astro-ph.SR]

[154] Ilias Cholis, Dan Hooper, and Tim Linden, "Constraining the Charge-Sign and Rigidity-Dependence of Solar Modulation," (2020), arXiv:2007.00669 [astro-ph.HE].

[155] http://www.srl.caltech.edu/ACE/ASC/,.

[156] http://wso.stanford.edu/Tilts.html,.

[157] M. Aguilar et al. (AMS), "Towards Understanding the Origin of Cosmic-Ray Positrons," Phys. Rev. Lett. 122, 
$041102(2019)$

[158] G. Ambrosi et al. (DAMPE), "Direct detection of a break in the teraelectronvolt cosmic-ray spectrum of electrons and positrons," Nature 552, 63-66 (2017). arXiv:1711.10981 [astro-ph.HE]

[159] O. Adriani et al., "Extended Measurement of the Cosmic-Ray Electron and Positron Spectrum from 11 $\mathrm{GeV}$ to $4.8 \mathrm{TeV}$ with the Calorimetric Electron Telescope on the International Space Station," Phys. Rev. Lett. 120, 261102 (2018), arXiv:1806.09728 [astroph.HE]

[160] P. Virtanen et al., "SciPy 1.0: Fundamental Algorithms for Scientific Computing in Python," Nature Methods 17, 261-272 (2020)

[161] Hans Dembinski and Piti Ongmongkolkul et al., "scikithep/iminuit," (2020), 10.5281/zenodo.3949207.

[162] J. Chang et al. (DAMPE), "The DArk Matter Particle Explorer mission," Astropart. Phys. 95, 6-24 (2017), arXiv:1706.08453 [astro-ph.IM].

[163] O. Adriani et al., "The CALorimetric Electron Telescope (CALET) for high-energy astroparticle physics on the International Space Station," in Journal of Physics Conference Series, Journal of Physics Conference Series, Vol. 632 (2015) p. 012023. 